\title{
Title: A large inner membrane pore defines the ESX translocon
}

Authors: Nicole Poweleit ${ }^{1,2}$, Nadine Czudnochowski ${ }^{1,2}$, Rachael Nakagawa ${ }^{1}$, Kenan Murphy ${ }^{3}$, Christopher Sassetti ${ }^{3}$, Oren S. Rosenberg ${ }^{1,2^{*}}$

\author{
Affiliations: \\ ${ }^{1}$ University of California, San Francisco, Department of Medicine, Division of Infectious \\ Diseases, San Francisco, CA 94158, USA. \\ ${ }^{2}$ Chan-Zuckerberg Biohub, University of California, San Francisco, CA 94158, USA. \\ ${ }^{3}$ Department of Microbiology and Physiological Systems, University of Massachusetts Medical \\ School, Worcester, MA, 01655, USA. \\ *Corresponding author. E-mail: oren.rosenberg@ucsf.edu
}

\section{Abstract:}

The ESX (or Type VII) secretion systems are protein export systems in mycobacteria and many Gram-positive bacteria that mediate a broad range of functions including virulence, conjugation, and metabolic regulation. These systems translocate folded dimers of WXG100superfamily protein substrates across the cytoplasmic membrane; however, the architecture and mechanism of translocation has remained elusive. We report the cryo-electron microscopy structure of an ESX-3 system, purified using an epitope tag inserted with recombineering into the model organism Mycobacterium smegmatis. The structure reveals two large $\alpha$-helical membrane pores of sufficient diameter to secrete folded substrates. A complex, asymmetric, multimeric cytoplasmic domain is poised to gate and regulate the pore's function. Our study provides mechanistic insights into the ESX systems and will guide structure-based design of drugs targeting this unique bacterial translocon.

One Sentence Summary: The structure of the ESX-3 secretion system reveals a pore of sufficient size for the transit of folded substrates and a complex, cytoplasmic regulatory apparatus. 
Main Text:

Mycobacteria use a set of specialized secretion systems called ESX to transport proteins

Mycobacteria tuberculosis (2-5), orthologs of ESX have since been discovered in most grampositive bacteria (6), and are more generally referred to as Type VII secretion systems (7). In mycobacteria there are five paralogous ESX operons (ESX 1-5) each of which encodes an inner membrane translocon complex consisting of three conserved Ecc proteins: EccB, EccC, and EccD. A fourth protein, EccE is conserved in all ESX operons except the ancestral ESX-4 operon and is also considered a part of the ESX translocon complex as it copurifies with EccB, EccC, and EccD (8). All Type VII secretion systems translocate proteins in the WXG100superfamily, which share a common two-helix hairpin structure and are found as homo- or heterodimers (9) and are mutually dependent for secretion with other substrates (10). In contrast to the general $(\mathrm{Sec})$ secretory apparatus, ESX substrates have been shown to be secreted in their folded, dimeric state (11).

Structural and functional information has been reported for truncated and isolated, soluble domains of the ESX translocon complexes and their homologs (12-15). A low resolution, negative stain electron microscopy structure of ESX-5 shows the translocon complex assembles into a hexamer (16). Structures of other proteins encoded in ESX operons including secreted substrates (17), substrate chaperons (18), and the regulator protease MycP (19) have been solved. Despite revealing important functional information about ESX, structures of overexpressed and isolated proteins are insufficient to understand how the translocation pore mediates the regulated secretion of fully folded substrates. We therefore undertook structural 
studies of a native ESX-3 complex from the model organism $M$. smegmatis using cryo-electron microscopy (cryo-EM).

The ESX-3 translocon complex is important for iron acquisition $(20,21)$, cell survival (22), and virulence in pathogenic mycobacteria (23), and its role in iron homeostasis is conserved in the model system, M. smegmatis. (20) The ESX-3 translocon complex proteins are transcribed in a single operon (24), and expression of the ESX-3 operon is dependent on the transcriptional regulator IdeR, which controls iron metabolism (25) and is required for growth in the human pathogen M. tuberculosis (26). The ESX-3 operon is $67 \%$ identical between the non-pathogenic model organism M. smegmatis and the pathogenic M. tuberculosis over the 4354 amino acids of the ESX-3 operon. This high degree of conservation and important role in cell growth makes ESX-3 an important candidate for small molecule inhibition(27), as blockade of ESX-3 will inhibit virulence and kill a broad range of pathogenic mycobacteria.

To facilitate native purification of the translocon complex, a cleavable GFP tag was inserted into the chromosome of M. smegmatis $\mathrm{MC}^{2} 155$ wild type and $\Delta i d e R(28)$ strains at the C-terminus of EccE $E_{3}$ via the ORBIT method (29) (Fig. 1A, Fig. S1). EccE $E_{3}$ is the final gene in the 11 gene long ESX-3 operon making insertion at this site less likely to disrupt regulation of the operon. Deletion of the iron acquisition regulator IdeR greatly increases chromosomal expression of ESX-3 (Fig. S2A). Four components of the complex EccB $3, \mathrm{EccC}_{3}, \mathrm{EccD}_{3}$ and $\mathrm{EccE}_{3}$ were affinity-purified as a large molecular weight species (Fig. 1B and 1C, Fig. S2B) using a GFP-nanobody and the GFP tag was proteolytically cleaved.

The ESX-3 translocon complex was imaged by cryo-EM and reconstructed revealing a dimeric structure which can be divided into four functionally important areas, the transmembrane translocon pore, the cytoplasmic translocon gate, the cytoplasmic motor domain, and the 
periplasmic multimerization domain (Fig. 1D, Fig. S3, Fig. S4, and Table S1). Particles of the size of a monomer or multimers larger than dimers were not observed in the electron microscopy data. The resolution of the ESX-3 translocon complex varies substantially in different parts of the electron microscopy map and this heterogeneity was partially resolved through data processing (see supplemental text, Fig. S5). The ESX-3 translocon complex is comprised of ten total proteins, two copies each of $\mathrm{EccB}_{3}, \mathrm{EccC}_{3}$, and $\mathrm{EccE}_{3}$ and four copies of $\mathrm{EccD}_{3}$. Two pseudo- symmetric protomers referred to as i and ii, combine to form the ESX-3 translocon complex. Each protomer contains one copy of $\mathrm{EccB}_{3}, \mathrm{EccC}_{3}$, and $\mathrm{EccE}_{3}$ and two conformationally distinct copies of $\mathrm{EccD}_{3}$, referred to as $\mathrm{EccD}_{3 \text {-front }}$ and $\mathrm{EccD}_{3 \text {-back }}$ (Fig. 2A). At $3.7 \AA$ resolution, the translocon pore and translocon gate were possible to build de novo atomic models for all observable amino acids except the two transmembrane helices of $\mathrm{EccC}_{3}$ (Fig. 2A, Table S2).

In each ESX-3 protomer, a distinct transmembrane pore is formed by the dimerization of the two copies of $\mathrm{EccD}_{3}, \mathrm{EccD}_{3 \text {-front }}$ and $\mathrm{EccD}_{3 \text {-back }}$ (Fig. 2B and 2C, Fig. S6A-C). The twentytwo $\mathrm{EccD}_{3} \alpha$-helices in each translocon pore form an elliptical, cylindrical void through the membrane with C2 symmetry and a cross-sectional diameter of $\sim 20$ x $30 \AA$ without significant regions of constriction (Fig. S6A). The inner surface of the translocon pores are composed primarily of hydrophobic residues (Fig. S6B) and in our maps, extended densities consistent with hydrophobic lipids or detergent molecules line the inner face of the pore (Fig. S6C). In contrast, the cytoplasmic face of the translocon pores are positively charged due to the presence of four basic residues (R134 and R239 from each EccD subunit) lining this region (Fig. S6B). Docking of the known structure of the dimeric $\mathrm{EsxG}_{3}-\mathrm{EsxH}_{3}(\mathrm{PDB} 3 \mathrm{Q} 4 \mathrm{H})$ (17) substrate into the 
translocon pores reveals an opening sufficient to allow for the transit of the fully folded substrate monomers and dimers (Fig. 2D-F).

The translocon gate is formed by interactions between the cytoplasmic domains of $\mathrm{EccD}_{3}$ -

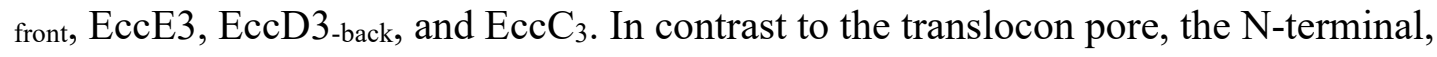
cytoplasmic domains of $\mathrm{EccD}_{3}$ are in strikingly different orientations, driven by alternative conformations of the residues joining the pore to the cytoplasmic domain (residues 100-127). This region of $\mathrm{EccD}_{3}$, adopts two distinct secondary structures resulting in the asymmetric

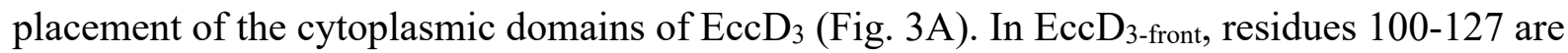
bent, folding into an $\alpha$-helix and forming extensive stabilizing contacts with $\mathrm{EccB}_{3}$ and $\mathrm{EccC}_{3}$ (Fig. S7A-G). In EccD 3 -back, residues 100-127 are extended and fold into a shorter $\alpha$-helix that interacts with $\mathrm{EccE}_{3}$ and the cytoplasmic domain of $\mathrm{EccD}_{3 \text {-front }}($ Fig. S7H). This conformational flexibility suggests that residues 100-127 may act as a hinge that changes its conformation during the secretion cycle, facilitating the movement of substrates from the cytoplasm to the translocation pores or blocking transit through the pores.

The next component of the translocon gate is $\mathrm{EccE}_{3} . \mathrm{EccE}_{3}$ is positioned at the front of the ESX-3 translocation complex, interacting with helix 11 of $\mathrm{EccD}_{3 \text {-front }}$ in the membrane and then extending into the cytoplasm (Fig. 3B and 3C, Fig. S8A-C). The cytoplasmic domain of $\mathrm{EccE}_{3}$ has weak structural homology to glycosyl transferase proteins, however, the nucleotide binding pocket is absent in $\mathrm{EccE}_{3}$ leaving it incapable of preforming this function (Fig. S8D and S8E, Table S4). Two helices in the cytoplasmic region of $\mathrm{EccE}_{3}$ between amino acids 133 and 163 form extensive stabilizing interactions with both subunits of $\mathrm{EccD}_{3}$ (Fig. S8F). These interactions hold the hinge region of $\mathrm{EccD}_{3 \text {-back }}$ in the extended conformation and sterically hinder $\mathrm{EccD}_{3 \text {-back }}$ from assuming the bent conformation seen in $\mathrm{EccD}_{3 \text {-front }}$ (Fig. S8G). 

$\mathrm{EccC}_{3} . \mathrm{EccC}_{3}$ extends from the membrane into the upper and lower cytoplasmic regions (Fig. 3D). Amino acids 1-33 and 94-403 of $\mathrm{EccC}_{3}$ were built de novo into the higher resolution region of the electron microscopy map revealing the structure of the DUF domain (Fig. 3E, Fig. S9AC).The de novo model of the DUF has the typical Rossman fold of a nucleotide hydrolysis domain, however, the Walker A motif contains substitutions and deletions that make ATP binding or hydrolysis by this domain unlikely (Fig. S9D and S9E). The protein binding helix remains intact and interacts with $\mathrm{EccD}_{3 \text { back }}$ (Fig. S9F) suggesting the DUF functions as a pseudoATPase in $\mathrm{EccC}_{3}(30)$. The pseudoATPase domain is linked to the transmembrane domains by a long helical bundle making extensive contacts with both the hinge region of $\mathrm{EccD}_{3 \text { front }}$ orienting the cytoplasmic domain of $\mathrm{EccD}_{3 \text { front }}$ asymmetrically (Fig. S7G). Neither the pseudoATPase domain of $\mathrm{EccC}_{3}$ nor the cytoplasmic domain of $\mathrm{EccE}_{3}$ appear capable of binding and hydrolyzing nucleotides. Instead, these proteins appear to collaborate to buttress the cytoplasmic domains of $\mathrm{EccD}_{3}$ and form gates in the cytoplasm positioned to regulate the translocon pores (Fig. 3F).

The motor domain of the ESX-3 translocon complex contains the $\mathrm{EccC}_{3}$ ATPase 1, 2 and 3, which hang below the pseudoATPase domain, were resolved at low resolution $\sim 10 \AA$, and are asymmetric between protomers i and ii (Fig. 3D, Fig. S9F and S9G). While both protomers have an identical protein composition, they differ in the conformations of their $\mathrm{EccB}_{3}$ and $\mathrm{EccC}_{3}$ components. When the translocation pore and gate are compared between protomers, only the transmembrane helices of $\mathrm{EccC}_{3}$ and the N-terminal tail of $\mathrm{EccB}_{3}$ differ (Fig. S10A and S10B). In contrast, the $\mathrm{EccC}_{3} \mathrm{ATPase} 1,2$, and 3 domains do not super impose accurately across 
protomers even at low resolution (Fig. S10C-E). The heterogeneity of the motor domains suggests this region is dynamic throughout the translocation cycle.

The ESX-3 translocon complex dimer is stabilized by cross-protomer interactions formed by the two $\mathrm{EccB}_{3}$ proteins which begin in the cytoplasm with a flexible N-terminal tail leading into a linker helix, followed by a single pass transmembrane helix, and an extended periplasmic domain (Fig. 3G and 3H, Fig. S11). The N-terminal tail of EccB 3 from protomer i forms extensive cross-protomer contacts with $\mathrm{EccB}_{3}, \mathrm{EccC}_{3}, \mathrm{EccD}_{3}$ front and $\mathrm{EccD}_{3}$ back from protomer ii (Fig. 3I, Table S3). The two $\mathrm{EccB}_{3}$ periplasmic domains share a large interaction interface across the protomers further stabilizing dimerization. Homology models of two $\mathrm{EccB}_{3}$ proteins can be docked into the periplasmic domain (Fig. S9C), however, this region is not resolved sufficiently to identify specific interactions. The majority of cross-protomer interactions involve $\mathrm{EccB}_{3}$ nucleotide. It is therefore likely to be in a conformation representing the end of the translocation cycle, awaiting either the direct binding of substrates or the binding of nucleotide to reset a substrate-binding competent state. The EsxB substrate is known to be recruited either as a homoor heterodimer in the cytoplasm by the ATPase 3 domain of EccC. Our structure is consistent with earlier reports that ESX substrates leave the translocation complex as folded dimers. The cytoplasmic domains of EccC, EccD, and EccE are idealy placed to regulate and guide substrates through the EccD pores (Fig. 4). The complexity of the ESX cytoplasmic assembly likely reflects the need for exquisite control of translocation in response to environmental changes and intracellular concentrations of substrates. 
Each protomer contains all the components required to translocate substrates across the inner membrane: the transmembrane translocation pore $(\mathrm{EccD})$, the cytoplasmic translocation gate (EccE, EccD, and EccC), and the cytoplasmic translocation motor domain (EccC). However, our structure of ESX-3 is clearly a dimer. The asymmetry of the EccC motor domains

Our structure of a type VII secretion system provides a roadmap for the further exploration of a ubiquitous class of secretion systems found in most gram-positive bacteria and provides a scaffold for drug discovery targeting mycobacterial ESX translocon complexes.

\section{References and Notes:}

Acknowledgments: The $\Delta \mathrm{IdeR}$ strain was provided by Gabriella Marcela Rodriguez. Data was collected at the electron microscopy core facility at the University of California, San Francisco with the assistance of Alexander G. Myasnikov. We thank Robert Stroud and the members of his laboratory for stimulating discussions.

Funding: Research reported here was supported by the NIH under award numbers 1RO1AI128214, 1U19AI135990-01, and P01AI095208. NP is funded in part by the NIH Host Pathogen Interaction training grant (5T32AI060537). Equipment in the UCSF EM facility used in this research was purchased with an NIH S10 grant (1S10OD021741). 
Competing interests: Authors declare no competing interests.

Data and materials availability: Electron microscopy micrographs are deposited on EMPIAR.

The electron microscopy maps are deposited to EMDB. The atomic models are deposited to the

PDB.

\section{List of Supplementary Materials:}

Materials and Methods

Supplemental Text

Figures S1-S11

Tables S1-S4

Movies S1-S\#

References (\#\#-\#\#)

Fig. 1. Overview of the ESX-3 tagging, purification, and structure. (A) The ESX-3 operon in

M. smegmatis and the placement of the purification tag. Genomic deletion of ideR derepresses

of all focused refinement maps (grey transparency) of the ESX-3 translocon complex filtered to $10 \AA$ resolution. The transmembrane and upper cytoplasmic focused maps $(3.7 \AA)$ segmented by 
subunit showing one copy per protomer of EccB3 (pink), EccC3 (blue), EccE3 (orange), EccD3front (yellow), EccD3-back (green).

Fig. 2. The translocon pore. (A) Atomic models of the translocon pore and gate. (B) Ecc $\mathrm{D}_{3 \text {-front }}$ (yellow) and EccD3-back (green) in the context of the overall ESX-3 translocon complex (grey transparency). (C) Atomic models of EccD 3 -front and $\mathrm{EccD}_{3 \text {-back }}$ (D) Space filling model of a single ESX-3 protomer with the substrate $\mathrm{EsxG}_{3}-\mathrm{EsxH}_{3}(\mathrm{PDB} 3 \mathrm{Q} 4 \mathrm{H})$ (grey) fitted into the pore.

Fig. 3. The translocon gate, motor, and multimerization domains. (A) EccD3-front (yellow) and EccD3-back (green) aligned based on the transmembrane regions shows two distinct conformations of the EccD3 cytoplasmic domains. Amino acids 100-127 of EccD3 adopt a bent (yellow) and an extended (green) conformation. (B) The placement of EccE3 in the overall ESX3 translocon complex. (C) Atomic model of EccE3 (D) The overall placement of EccC3 in the ESX-3 translocon complex. EccC3 was reconstructed to variable resolutions. The upper cytoplasmic pseudoATPase domain (aa 1-37 and 94-403) in the translocation gate was reconstructed to 3.7 $\AA$. The lower cytoplasmic, ATPase 1, 2, and 3 domains in the translocon motor were reconstructed to $\sim 10 \AA$ resolution. (E) Atomic model of the EccC3 psuedoATPase domain. (F) Atomic model of the translocon gate comprised of EccE3 (orange), the cytoplasmic domain of EccD3-front (yellow), the cytoplasmic domain of EccD3-back (green), and the pseudoATPase domain of EccC3 (blue). (G) EccB3 (pink) in the context of the overall ESX-3 translocon complex (grey transparency). EccB3 has a single pass transmembrane domain which extends into a large periplasmic domain which was resolved at $5.5 \AA$ resolution. $(\mathrm{H})$ Atomic models of the EccB3 cytoplasmic and transmembrane domains, amino acids 14-93 and 32-93. (I) The N-terminus of EccB3 forms extensive cross-protomer contacts with EccC3 (blue), EccD3front (yellow), and EccD3-back (green). 
225 Fig. 4. Hybrid model of the ESX-3 translocon complex. (A) A combined map of the full 226 complex filtered to $10 \AA$ resolution (grey transparency) with models for each protein, EccB3

227 (pink), EccD3-front (yellow), EccD3-back (green), EccC3 (blue), and EccE3 (orange). (B) A 228 model of the function of a single protomer of the ESX-3 complex. Substrates are selected by 229 interaction with ATPase 3 of EccC and transported via the upper cytoplasmic gate to the 230 translocon pore for secretion. 
Bibliography

1. M. I. Gröschel, F. Sayes, R. Simeone, L. Majlessi, R. Brosch, ESX secretion systems: mycobacterial evolution to counter host immunity. Nat. Rev. Microbiol. 14, 677-691 (2016).

2. S. A. Stanley, S. Raghavan, W. W. Hwang, J. S. Cox, Acute infection and macrophage subversion by Mycobacterium tuberculosis require a specialized secretion system. Proc. Natl. Acad. Sci. U. S. A. 100, 13001-13006 (2003).

3. K. N. Lewis et al., Deletion of RD1 from Mycobacterium tuberculosis mimics bacille Calmette-Guérin attenuation. J. Infect. Dis. 187, 117-123 (2003).

4. K. M. Guinn et al., Individual RD1-region genes are required for export of ESAT-6/CFP10 and for virulence of Mycobacterium tuberculosis. Mol. Microbiol. 51, 359-370 (2004).

5. T. Hsu et al., The primary mechanism of attenuation of bacillus Calmette-Guerin is a loss of secreted lytic function required for invasion of lung interstitial tissue. Proc. Natl. Acad. Sci. USA. 100, 12420-12425 (2003).

6. D. Bottai, M. I. Gröschel, R. Brosch, Type VII Secretion Systems in Gram-Positive Bacteria. Curr. Top. Microbiol. Immunol. 404, 235-265 (2017).

7. W. Bitter et al., Systematic genetic nomenclature for type VII secretion systems. PLoS Pathog. 5, e1000507 (2009).

8. E. N. G. Houben et al., Composition of the type VII secretion system membrane complex. Mol. Microbiol. 86, 472-484 (2012).

9. C. Poulsen, S. Panjikar, S. J. Holton, M. Wilmanns, Y.-H. Song, WXG100 protein superfamily consists of three subfamilies and exhibits an $\alpha$-helical C-terminal conserved residue pattern. PLoS One. 9, e89313 (2014).

10. S. M. Fortune et al., Mutually dependent secretion of proteins required for mycobacterial virulence. Proc. Natl. Acad. Sci. USA. 102, 10676-10681 (2005).

11. T. A. Sysoeva, M. A. Zepeda-Rivera, L. A. Huppert, B. M. Burton, Dimer recognition and secretion by the ESX secretion system in Bacillus subtilis. Proc. Natl. Acad. Sci. USA. 111, 7653-7658 (2014).

12. X.-L. Zhang et al., Core component EccB1 of the Mycobacterium tuberculosis type VII secretion system is a periplasmic ATPase. FASEB J. 29, 4804-4814 (2015).

13. O. S. Rosenberg et al., Substrates Control Multimerization and Activation of the MultiDomain ATPase Motor of Type VII Secretion. Cell. 161, 501-512 (2015).

14. M. Zoltner et al., EssC: domain structures inform on the elusive translocation channel in the Type VII secretion system. Biochem. J. 473, 1941-1952 (2016).

15. J. M. Wagner et al., Structures of EccB1 and EccD1 from the core complex of the mycobacterial ESX-1 type VII secretion system. BMC Struct Biol. 16, 5 (2016).

16. K. S. H. Beckham et al., Structure of the mycobacterial ESX-5 type VII secretion system membrane complex by single-particle analysis. Nat. Microbiol. 2, 17047 (2017).

17. D. Ilghari et al., Solution structure of the Mycobacterium tuberculosis EsxG·EsxH complex: functional implications and comparisons with other M. tuberculosis Esx family complexes. J. Biol. Chem. 286, 29993-30002 (2011).

18. D. C. Ekiert, J. S. Cox, Structure of a PE-PPE-EspG complex from Mycobacterium tuberculosis reveals molecular specificity of ESX protein secretion. Proc. Natl. Acad. Sci. USA. 111, 14758-14763 (2014). 
19. M. Solomonson et al., Structure of the mycosin-1 protease from the mycobacterial ESX-1 protein type VII secretion system. J. Biol. Chem. 288, 17782-17790 (2013).

20. M. S. Siegrist et al., Mycobacterial Esx-3 is required for mycobactin-mediated iron acquisition. Proc. Natl. Acad. Sci. USA. 106, 18792-18797 (2009).

21. A. Serafini, D. Pisu, G. Palù, G. M. Rodriguez, R. Manganelli, The ESX-3 secretion system is necessary for iron and zinc homeostasis in Mycobacterium tuberculosis. PLoS One. 8, e 78351 (2013).

22. E. Tinaztepe et al., Role of Metal-Dependent Regulation of ESX-3 Secretion in Intracellular Survival of Mycobacterium tuberculosis. Infect. Immun. 84, 2255-2263 (2016).

23. J. M. Tufariello et al., Separable roles for Mycobacterium tuberculosis ESX-3 effectors in iron acquisition and virulence. Proc. Natl. Acad. Sci. USA. 113, E348-57 (2016).

24. X. Li et al., Transcriptome Landscape of Mycobacterium smegmatis. Front. Microbiol. 8, 2505 (2017).

25. G. M. Rodriguez, M. I. Voskuil, B. Gold, G. K. Schoolnik, I. Smith, ideR, An essential gene in mycobacterium tuberculosis: role of IdeR in iron-dependent gene expression, iron metabolism, and oxidative stress response. Infect. Immun. 70, 3371-3381 (2002).

26. R. Pandey, G. M. Rodriguez, IdeR is required for iron homeostasis and virulence in Mycobacterium tuberculosis. Mol. Microbiol. 91, 98-109 (2014).

27. D. Bottai, A. Serafini, A. Cascioferro, R. Brosch, R. Manganelli, Targeting type VII/ESX secretion systems for development of novel antimycobacterial drugs. Curr. Pharm. Des. 20, 4346-4356 (2014).

28. O. Dussurget, M. Rodriguez, I. Smith, An ideR mutant of Mycobacterium smegmatis has derepressed siderophore production and an altered oxidative-stress response. Mol. Microbiol. 22, 535-544 (1996).

29. K. C. Murphy et al., ORBIT: a new paradigm for genetic engineering of mycobacterial chromosomes. MBio. 9 (2018), doi:10.1128/mBio.01467-18.

30. P. A. Eyers, J. M. Murphy, The evolving world of pseudoenzymes: proteins, prejudice and zombies. BMC Biol. 14, 98 (2016).

31. V. J. C. van Winden et al., Mycosins Are Required for the Stabilization of the ESX-1 and ESX-5 Type VII Secretion Membrane Complexes. MBio. 7 (2016), doi:10.1128/mBio.01471-16. 


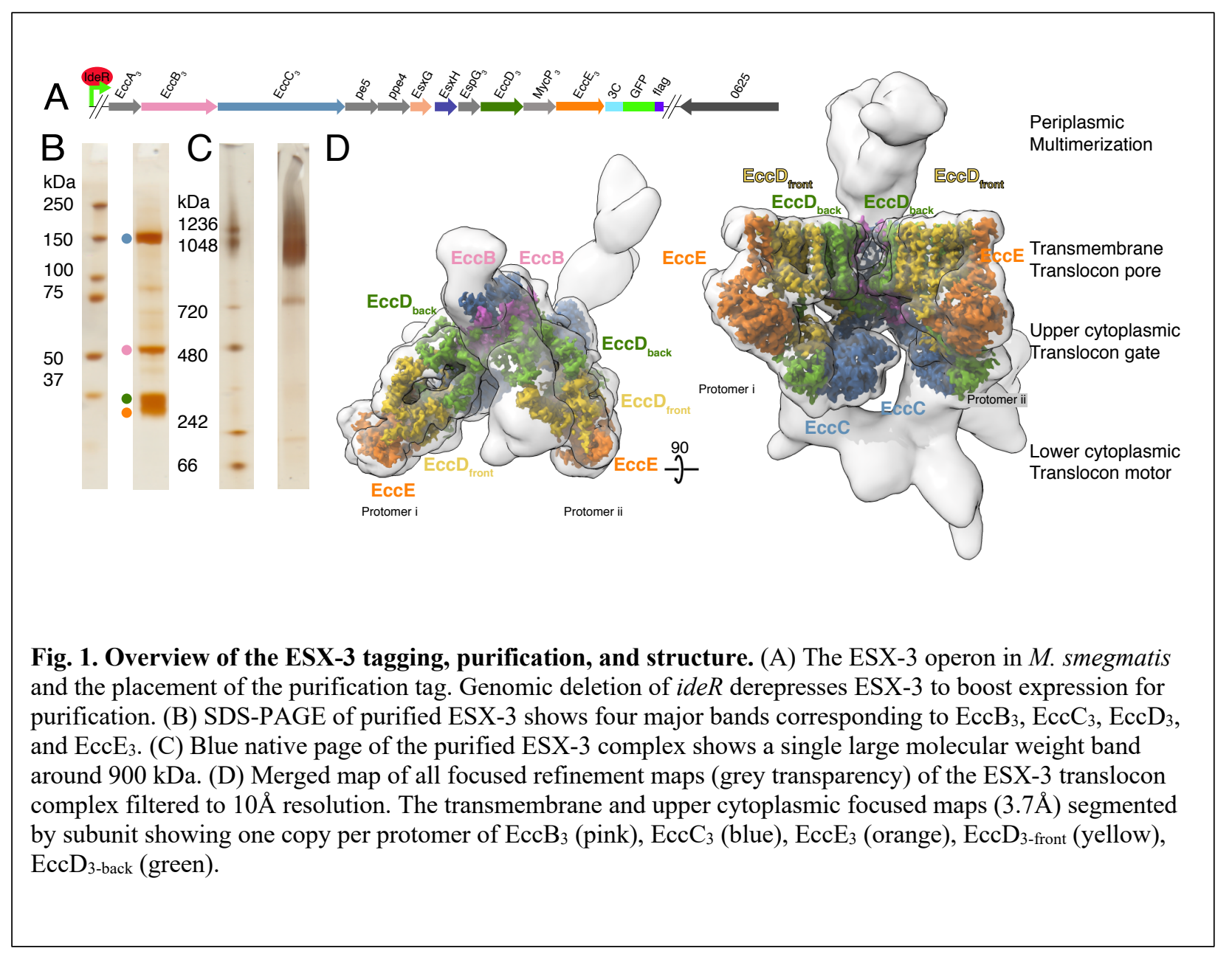



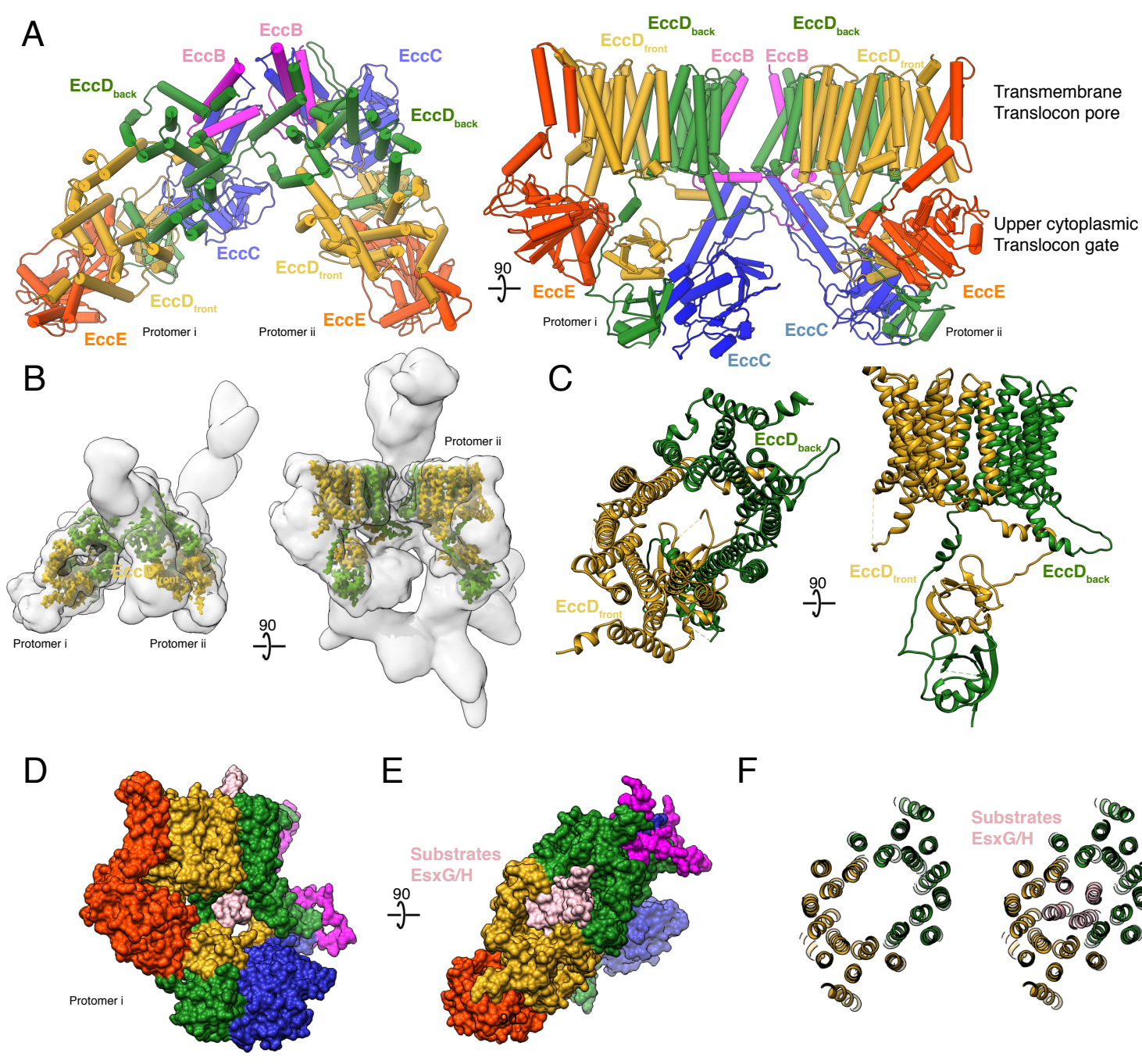

$\mathrm{F}$

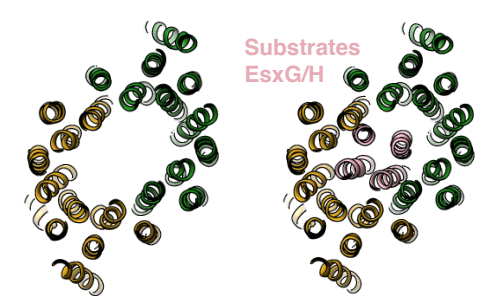

Fig. 2. The translocon pore. (A) Atomic models of the translocon pore and gate. (B) EccD3-front (yellow) and EccD3-back (green) in the context of the overall ESX-3 translocon complex (grey transparency). (C) Atomic models of EccD3-front and EccD3-back (D) Space filling model of a single ESX-3 protomer with the substrate $\mathrm{EsxG}_{3}-\mathrm{EsxH}_{3}$ (PDB 3Q4H) (grey) fitted into the pore. 


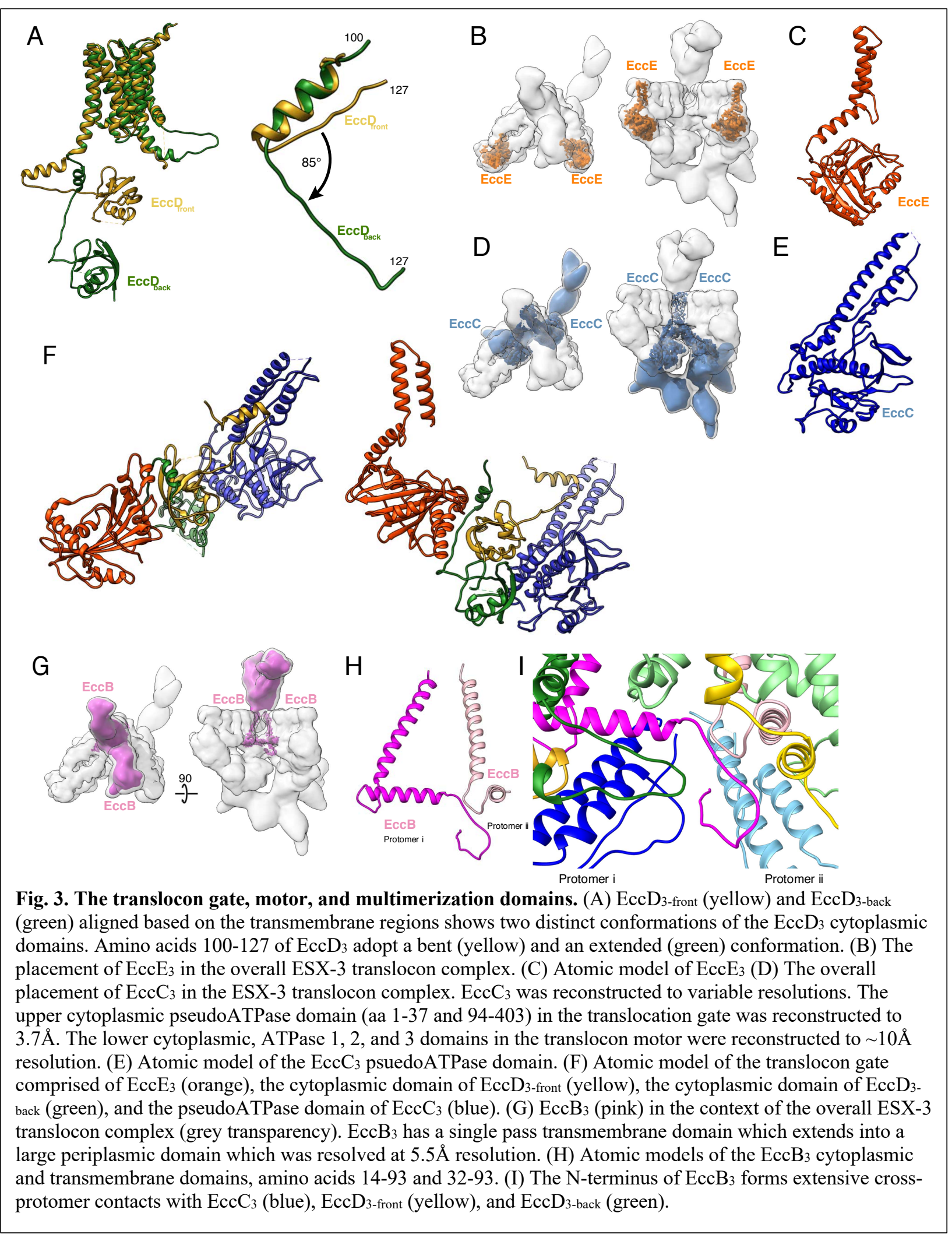




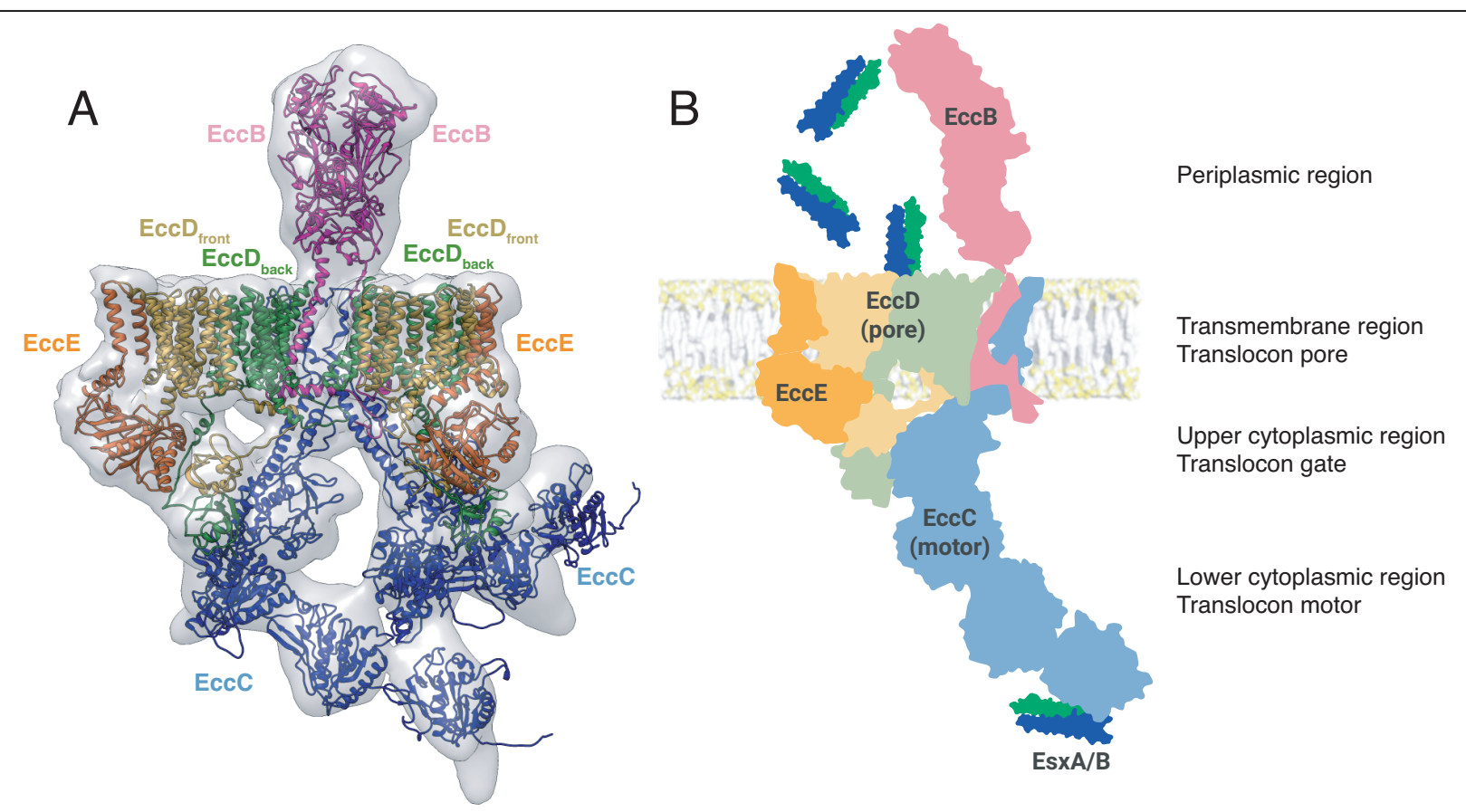

Fig. 4. Hybrid model of the ESX-3 translocon complex. (A) A combined map of the full complex filtered to $10 \AA$ resolution (grey transparency) with models for each protein, $\mathrm{EccB}_{3}$ (pink), $\mathrm{EccD}_{3 \text {-front }}$ (yellow), Ecc $\mathrm{D}_{3 \text {-back }}$ (green), $\mathrm{EccC}_{3}$ (blue), and $\mathrm{EccE}_{3}$ (orange). (B) A model of the function of a single protomer of the ESX-3 complex. Substrates are selected by interaction with ATPase 3 of EccC and transported via the upper cytoplasmic gate to the translocon pore for secretion. 


\title{
S \\ Supplementary Materials for:
}

Title: A large inner membrane pore defines the translocation pathway of ESX substrates

This PDF file includes:

$\underline{\text { Strain construction }}$

Materials and Methods

Supplementary Text

Figs. S1 to S11

Tables S1 to S4

\section{Materials and Methods}

\author{
Correspondence to: oren.rosenberg@ucsf.edu
}

Christopher Sassetti $^{1}$, Nadine Czudnoch S. Rosenberg ${ }^{*}$
Christ $^{1}$, Rachael Nakagar 
31 were plated on hygromycin containing $7 \mathrm{H} 9$ plates and incubated at $37^{\circ} \mathrm{C}$ for 3 days. Colonies

32 were verified for insertion of the tagging plasmid into the chromosome by PCR.

$\underline{\text { Western Blotting }}$

$100 \mathrm{~mL}$ of $\mathrm{EccE}_{3}$ tagged wild type and IdeR knock out cells were grown overnight to an

OD of 1.0-1.2. Cells were pelleted and resuspended in $1 \mathrm{ml}$ of buffer $(50 \mathrm{mM}$ Tris-HCl $\mathrm{pH} 8.0$, $150 \mathrm{mM} \mathrm{NaCl}, 1 \% \mathrm{DDM}$ ) and sonicated for 30 seconds. Cell lysates were run on a 4-20\% SDS-

PAGE gel (Genescript) and transferred to blotting paper using a BioRad Trans-blot turbo system.

38 The blot was washed with PBS and blocked in a 5\% milk solution for 1 hour. The blot was

39 incubated with mouse anti-GFP monoclonal antibody (Roche) overnight. After rinsing with PBS-

$40 \mathrm{~T}$, the blot was incubated with anti-mouse IgG HRP-conjugated antibody (R\&D Systems) for 2

41 hours. After activation (Amersham) the blot was imaged on a BioRad ChemiDoc. The blot was

42 stripped with stripping buffer (ThermoFisher Scientific) as per the manufactures instructions, and

43 incubated overnight with rabbit anti-GroEL monoclonal antibody (Sigma-Aldrich). The blot was

44 incubated with goat anti-rabbit IgG antibody HRP (GenScript) for 2 hours, activated

45 (Amersham), and imaged on a BioRad ChemiDoc.

Protein purification

M. smegmatis was grown in $7 \mathrm{H} 9$ supplemented with $0.05 \%$ Tween 80 and $20 \mu \mathrm{g} / \mathrm{ml}$ kanamycin. After harvest, cells were washed three times with PBS and frozen in liquid nitrogen

49 before lysis with a cryogenic grinder (SPEX SamplePrep). 24.9g of powdered cell material was

50 resuspended in $56.3 \mathrm{ml} 50 \mathrm{mM}$ Tris- $\mathrm{HCl} \mathrm{pH} 8.0,150 \mathrm{mM} \mathrm{NaCl}, 1 \%$ DDM with protease

51 inhibitor cocktail (SigmaFast) and 175 units Benzonase endonuclease. The suspension was 
52 stirred for $120 \mathrm{~min}$ at $4^{\circ} \mathrm{C}$. After centrifugation for $30 \mathrm{~min}$ at $98,000 \mathrm{~g}$ (ThermoLynx), the

53 supernatant was incubated with $1.4 \mathrm{ml}$ anti-GFP-nanobody resin for $90 \mathrm{~min}$ at $4^{\circ} \mathrm{C}$. The resin was

54 transferred to a column and washed sequentially with $28 \mathrm{ml}$ of wash buffer $(50 \mathrm{mM}$ Tris- $\mathrm{HCl} \mathrm{pH}$

$558.0,150 \mathrm{mM} \mathrm{NaCl}$ and $0.1 \% \mathrm{GDN}), 14 \mathrm{ml}$ of high salt wash buffer (50 mM Tris-HCl $\mathrm{pH} 8.0,400$

$56 \mathrm{mM} \mathrm{NaCl}$, and $0.1 \% \mathrm{GDN})$, and $14 \mathrm{ml}$ of wash buffer $(50 \mathrm{mM}$ Tris- $\mathrm{HCl} \mathrm{pH} 8.0,150 \mathrm{mM} \mathrm{NaCl}$,

57 and $.1 \% \mathrm{GDN}$ ). To cleave off the purification tag, the resin was incubated $\mathrm{o} / \mathrm{n}$ at $4{ }^{\circ} \mathrm{C}$ with 70

58 units Pierce HRV 3C protease (ThermoFisher Scientific) in $2.8 \mathrm{ml}$ wash buffer supplemented

59 with $0.2 \mathrm{mM}$ DTT. This resin was sedimented by gentle centrifugation ( $300 \mathrm{xg}$ for $3 \mathrm{~min})$, the

60 supernatant collected, and the resin was subsequently washed with $1.4 \mathrm{ml}$ wash buffer. The

61 supernatant and first wash fraction were combined and concentrated using an Amicon Ultra-4

62 centrifugal filter unit (100 kDa molecular weight cut-off). The sample was centrifuged for 6 min

63 at $16,000 \mathrm{~g}$ before injection on a Superdex $200(10 / 300)$ column equilibrated in $50 \mathrm{mM}$ Tris- $\mathrm{HCl}$

$64 \mathrm{pH} 8.0,150 \mathrm{mM} \mathrm{NaCl}$ and $0.021 \%$ GDN. Peak fractions were concentrated using a $0.5 \mathrm{ml}$

65 centrifugal filter unit (Amicon, $100 \mathrm{kDa}$ cut-off) to $5.52 \mathrm{mg} / \mathrm{ml}$ by Nanodrop reading.

66 Blue-Native Polyacrylamide Gel Electrophoresis (BN-PAGE)

BN-PAGE was carried out using the Invitrogen NativePAGE Bis-Tris Gel system.

68 Samples were prepared in a total volume of $10 \mu \mathrm{l}$ using $0.25 \mu 15 \%$ G-250 sample additive.

69 Electrophoresis was performed using light blue cathode buffer at a constant voltage of $\sim 105 \mathrm{~V}$

70 for $3.5 \mathrm{~h}$ at $4^{\circ} \mathrm{C}$. The gel was fixed and stained using the Pierce silver stain kit.

71 Cryo electron microscopy - data acquisition 
Samples were frozen for cryo electron microscopy. Quantifoil R1.2/1.3, 400 mesh, cooper grids were glow discharged using a Solarus plasma cleaner (Gatan) with an $\mathrm{H}_{2} / \mathrm{O}_{2}$

74 mixture for 30 seconds. $2 \mu 1$ of sample were applied per grid and the grids were plunged into

75 liquid ethane using a FEI Vitrobot Mark IV.

Initially, samples were screened, and test data sets were collected on a FEI Talos Arctica 200kV microscope equipped with a Gatan K2 Summit detector. For the initial screen of freezing conditions, 834 movies were collected at a magnification of 36,000 with a pixel size of 1.14 , and a defocus range of -1.5 to $-2.5 \mu \mathrm{m}$, an exposure time of 9 seconds, and a dose rate of 7 electrons $/ \AA^{2} /$ second. (Table S1). Data collection for the final structure presented in the main text

81 was collected on a FEI Titan Krios at 300kV with a Gatan K2 Summit detector. Two imaging sessions were used. In the first imaging session, 2705 movies were collected at a magnification

83 of 29,000 with a pixel size of 0.82 , and a defocus of -0.4 to $-1.2 \mu \mathrm{m}$, an exposure time of 10

84 seconds to collect 100 total frames, and a dose of 8 electrons $/ \AA^{2} /$ second. (Table $\mathrm{S} 1$ ). In the second imaging session, data was collected on the same microscope with the same detector, 4632

86 movies were collected at a magnification of 29,000 with a pixel size of 0.82 , and a defocus range

87 of -0.6 to $-1.4 \mu \mathrm{m}$, an exposure time of 10 seconds to collect 80 total frames, and a dose of 6.7

88 electrons $/ \AA^{2} /$ second.

For all data, movies were motion corrected using MotionCor2(1) and CTF correction was

91 performed using CTFfind4(2). For the Arctica dataset, particles were picked using a gaussian

92 blob in either RELION(3) or cisTEM(4) and initial 2D classification was performed to remove

93 obvious junk particles. Initially, a shotgun approach was taken to generate several initial models 
94 using RELION, cisTEM, and cryosparc(5). Once an initial model which contained realistic low-

95 resolution features was generated, a user defined descent gradient was performed to improve the

96 model with the goal of achieving accurate secondary structure features. First, all particles

97 selected during 2D classification were refined in 3D against the randomly generated initial

98 model. Second, a round of 3D classification with 4 classes and default RELION settings was

99 performed and the best class selected. Third, the best class was refined as a single class in 3D

100 classification with increasing Tau2_Fudge and decreasing search angle size. The resulting EM

101 density map had clear transmembrane helix densities and was used as the model for a new 3D

102 reconstruction. This reconstruction was used to back project models for reference-based particle

103 picking in RELION. Two rounds of 2D classification were performed and the best classes

104 selected. One round of 3D classification was performed using the Tau2_Fudge value optimized

105 during the previous run through $(\mathrm{T}=12)$ and the best class selected. A final 3D reconstruction of

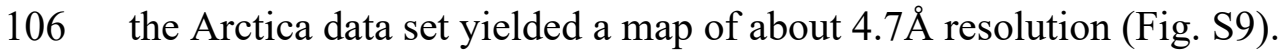

108 processed entirely using RELION. Particles were picked using a gaussian blob, and extracted as

$1094 \mathrm{x}$ binned particles. Two rounds of initial $2 \mathrm{D}$ classification were performed with $\mathrm{T}=3$ on the

110 binned particles and obvious junk particles were removed. The final reconstruction from the

111 Arctica dataset was used as the initial model for a 3D reconstruction of the binned particles. 3D

112 classification with 4 classes and the previously optimized Tau2_Fudge value, $\mathrm{T}=12$, was

113 performed on the binned particles. The two best class were selected and re-extracted without

114 binning. A 3D reconstruction was performed. A mask was created for the high-resolution region

115 of the reconstruction and 3D classification without image alignment was performed focused on

116 this region. The best class was selected and the subsequent $4.0 \AA$ reconstruction is the consensus 
117 structure for the entire complex (Fig. S3). Focused classification of each protomer, the

118 periplasmic EccB region, and the ATPase 1,2, and 3 domains of EccC were performed. To

119 perform focused classification, the center of mass of the region of interest was determined using

120 chimera $(6)$. Particles were recentered on this area and reextracted. Masks for the region of

121 interest were generated and 3D classification without image alignment was performed. The best

122 class was selected and used for a focused 3D reconstruction without image alignment of the

123 region of interest. A reconstruction was generated and density outside of the region of interest

124 was subtracted. A final reconstruction of the masked and density subtracted particles was then

125 performed. This procedure improved the resolution of the protomers to $3.75 \AA$ (left) and $3.83 \AA$

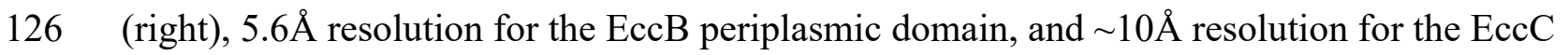

127 lower cytoplasmic region.

To generate the symmetry expanded protomers based on non-point group symmetry (also

129 known as non-crystallographic symmetry or NCS), a transformation matrix between the two

130 protomers was calculated using chimera. Particles were then transformed and aligned using the

131 subparticles.py and star.py utilities in pyem[cite] resulting in a particle stack with twice as many

132 particles as the input file, each focused on protomer i or protomer ii. Density subtraction was

133 performed to remove density outside of the symmetry expanded protomer, and focused

134 classification and refinement were performed as described above. This procedure improved the

135 resolution of the symmetry expanded protomer to $3.69 \AA$ resolution.

136 Atomic model building

137 The cytoplasmic domain from the crystal structure of EccD 1 (PDB 4KV2) was docked

138 into the cytoplasmic domains of the two $\mathrm{EccD}_{3}$ molecules and the sequence was mutated. The 
139 remaining transmembrane domains of $\mathrm{EccD}_{3}$ and the residues $14-93$ of $\mathrm{EccB}_{3}$ were built de novo

140 in $\operatorname{Coot}(7)$ using baton building. The alpha helices of $\mathrm{EccE}_{3}$ and $\mathrm{EccC}_{3}$ were initially modeled

141 using the RaptorX(8) homology server. The loops and strands of $\mathrm{EccE}_{3}$ and $\mathrm{EccC}_{3}$ were built in

142 Coot using baton building. All models were subsequently refined individually, as a symmetry

143 expanded protomer, left and right protomers, and as the full model using phenix real space

144 refine(9), Coot, and the $\operatorname{MDFF}(10)$ server, namdinator(11) (Table S2).

145 Low resolution modeling

146 The left and right protomer map, periplasmic focused refined map, and lower cytoplasmic

147 focused refined map were all docked into the consensus map and added together using chimera.

148 The combined map was filtered to $10 \AA$ resolution to match the lowest resolution component.

149 Homology models for amino acids $94-516$ of $\mathrm{EccB}_{3}$, the transmembrane helixes of $\mathrm{EccC}_{3}$, and

150 404-1268 of EccC $_{3}$ were generated using RaptorX. These models were fit into the combined map

151 density using the fit map to model utility in Chimera. The full model was refined using

152 phenix.real_space_refine.

$153 \quad$ Model interpretation and display

154 The pore diameter and properties were analyzed using MOLE(12). Buried surface area

155 between subunits was calculated by PISA(13). Atomic models for individual proteins were

156 compared against the PDB using the DALI server(14). Chimera and chimerax(15) were used to

157 display maps and models for figure creation. 


\section{Supplementary Text}

Initially, the entire ESX-3 translocon complex was reconstructed to $4.0 \AA \AA$ resolution (Fig.

160 S5A). Using symmetry expansion, and focused classification and refinement techniques, the

161 resolutions of targeted regions of the ESX-3 complex were improved to $3.7 \AA$ for the

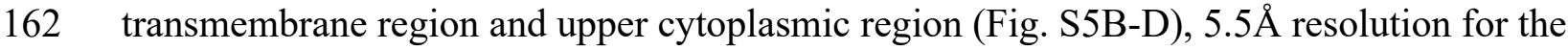

163 periplasmic region (Fig. S5E), and $10 \AA \AA$ resolution for the lower cytoplasmic region (Fig. S5F).

164 The highest resolution maps for each region were combined and filtered to the threshold of the

165 lowest resolution map to form an overall $10 \AA$ consensus map for the entire translocon complex

166 The front EccD molecule forms the following interactions: Arg11 of the front EccD

167 forms an ionic interaction with Glu161 of EccE. Asp14 of the front EccD forms an ionic

168 interaction with Arg99 of EccE. Arg23 of the front EccD forms an ionic interaction with Asp111

169 of the back EccD. Glu26 of the front EccD forms an ionic interaction with Asp157 of EccE.

170 Glu32 of the front EccD forms backbone contacts with Gly18 and Asp19 of the back EccD.

171 Arg36 of the front EccD interacts with Glu389 of EccC. Pro40 of the front EccD interacts with

172 His392 of EccC. Glu37 of the front EccD interacts with Arg395 of EccC. Arg66 of the front

173 EccD interacts with Glu134 and Glu389 of EccC. Arg107 of the front EccD interacts with

174 Arg116 of EccC. Glu 110 of the front EccD interacts with Arg112 of EccC. Asp111 and Asp114

175 of the front EccD interact with Arg47 of EccB. Phe119 of the front EccD interacts with Pro321

176 of the back EccD. Ser120 of the front EccD interacts ionically with Gln328 of the back EccD and

177 His55 of EccB. Glu121 of front EccD interacts ionically with His55 of EccB. Arg123 of the front

178 EccD interacts with Arg239 and Arg325 of the back EccD. Arg124 of the front EccD interacts

179 with Asp378 of the back EccD and His55 of EccB. Arg125, His131, and Arg134 of the front

180 EccD are coordinating a non-protein density together. Gln126 of the front EccD is interacting 
181 with Asp378 of the back EccD. Trp127 of the front EccD has a cation- $\pi$ interaction with Lys383

182 of the back EccD. Pro129 of the front EccD has an interaction with Pro431 of the back EccD.

183 The residues in transmembrane helix 1 of the front EccD have several non-specific hydrophobic

184 interactions with transmembrane helices 9 and 10 of the back EccD. These last three interactions

185 between the EccD front and back protein's Trp127 and Lys383, Pro129 and Pro431, and

186 transmembrane helix 1 and transmembrane helices 9 and 10 are mirrored on the other side of the

187 pore. Transmembrane helix 11 of the front EccD forms non-specific hydrophobic interactions

188 with transmembrane helix 1 of EccE.

The back EccD forms the following interactions: Arg11 of the back EccD protrudes into

190 a negatively charged pocket on EccC formed by Asp268, Ser398, and Asp401. Gly18 and Asp19

191 of the back EccD have backbone interactions with Glu32 of the front EccD. Glu26 of the back

192 EccD forms an ionic interaction with Arg395 of EccC. Asp92 of the back EccD interacts with

193 Arg399 of EccC. Ala105 of the back EccD forms a backbone contact with Glu161 of EccE.

194 Glu110 of the back EccD forms an ionic contact with Arg138 and Arg154 of EccE. Between

195 Ile108 and Ile112 of the back EccD is a beta strand which complements the beta sheet of the

196 cytoplasmic domain of the front EccD. Asp111 of the back EccD forms an ionic interaction with

197 Arg23 from the front EccD.

198 Of the 80 amino acids modeled in EccB, almost all are involved in important inter or

199 intra molecular interactions. Arg16 of EccB forms ionic contacts with Asp178 and Glu179 of

200 EccC from the same protomer and could potentially from an ionic contact with Tyr107 of EccC

201 from the opposite protomer. Thr17 of EccB forms an ionic contact with Arg106 of EccC from

202 the opposite protomer. Asn20 of EccB forms an ionic contact with Asp113 of EccC from the

203 opposite protomer. Glu21 of EccB forms an ionic contact with Asn114 of EccC from the 
opposite protomer. Asn22 of EccB forms an ionic contact with Asp113 of EccC from the opposite protomer. Asp24 of EccB forms an ionic contact with Arg116 of EccC from the opposite protomer. The side chain position of Arg29 of EccB is ambiguous and it could form an ionic contact with Asp306 of the back EccD molecule from the same protomer or Asp378 of the back EccD molecule from the opposite protomer. The side chain position of $\mathrm{Arg} 30$ of EccB is ambiguous and it could form ionic contact with Asp378 or Asp437 of the back EccD molecule from the opposite protomer. Phe32 of EccB forms a cation- $\pi$ interaction with Arg375 of the back

211 EccD from the opposite protomer. Arg35 of EccB forms an interaction with Arg445 of the back

212 EccD from the opposite protomer. His36 of EccB might form an ionic interaction with Asp181

213 of EccC from the same protomer. Gln37 of EccB forms an ionic interaction with Asp306 from

214 the back EccD from the same protomer. Ser39 of EccB forms an ionic interaction with Arg101

215 of EccC from the same protomer. Trp41 of EccB forms $\mathrm{CH} / \pi$ interactions with Pro442 and

216 Pro229 from the back EccD from the same protomer. Arg42, Arg46, Arg65, and Arg69 of EccB

217 all interact with each other to stabilize the loop between the linker and transmembrane helix of

218 EccB. All four of these arginine residues may also interact with Asp98 of EccC from the same

219 protomer. This interaction may also be involved in coordinating lipids or locally melting the

220 membrane to allow for flexibility. Phe43 of EccB forms a cation- $\pi$ interaction with Arg101 of

221 EccC from the same protomer. Arg47 of EccB forms ionic contacts with Asp111 and Asp114

222 from the front EccD from the same protomer. His 55 of EccB sits in a pocket of charged amino

223 acids and participates in a complex interaction network with Glu121 and Arg124 from the front

224 EccD and Asp378 from the back EccD all from the same protomer. Arg58 of EccB interacts with

225 Arg106 of EccC from the same protomer. The transmembrane helix of EccB, Pro63 to Phe91,

226 forms several non-specific hydrophobic interactions with the back EccD transmembrane helix 11 
227 in the same protomer. Overall, EccB forms a substantial number of protein-protein interactions

228 and all substantial cross-protomer interactions involve EccB making it essential for

229 multimerization of ESX-3 protomers.

230 
A

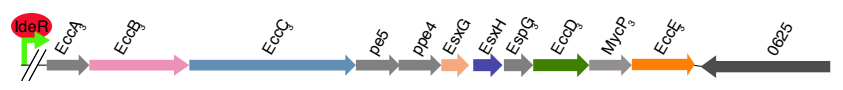

$\mathrm{B}_{5}$ Downstream homology AttP site Upstream homology 5' TGTGCGTTCCACTGGTTCCCCGGCAACCACCTGCTGCACGTGAGCCAGCCGGACTACCTAGGTTGTACCGTACACCACTGAGACCGCGGTGGTTGACCAGACAAACCCGCCGGATGACCCGCTTCCTGCGCGGCTTCA

\section{C}
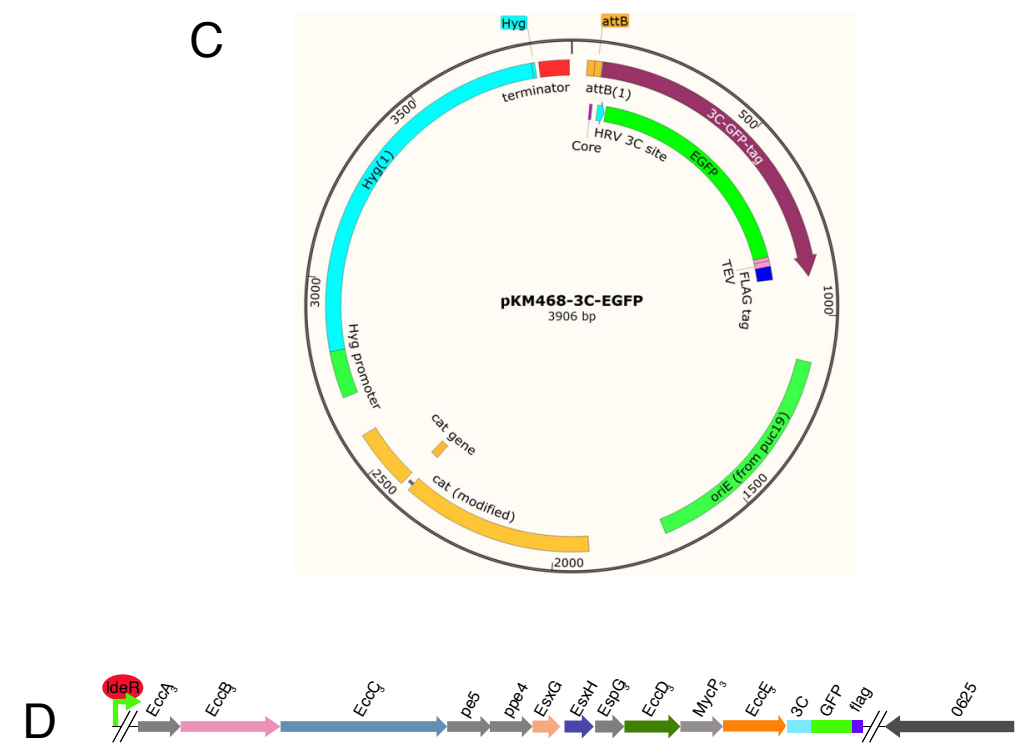

\section{Fig. S1. ORBIT tagging of the chromosomal copy of $\mathbf{E c c E}_{3}$}

234 (A) Cartoon of the ESX-3 operon present in the M. smegmatis MC 155 chromosome. (B) The targeting oligo used to insert the attp site into the chromosome. (C) The tagging plasmid recombined into the chromosome. (D) Cartoon of the resulting change to the chromosome after the ORBIT protocol was performed. 


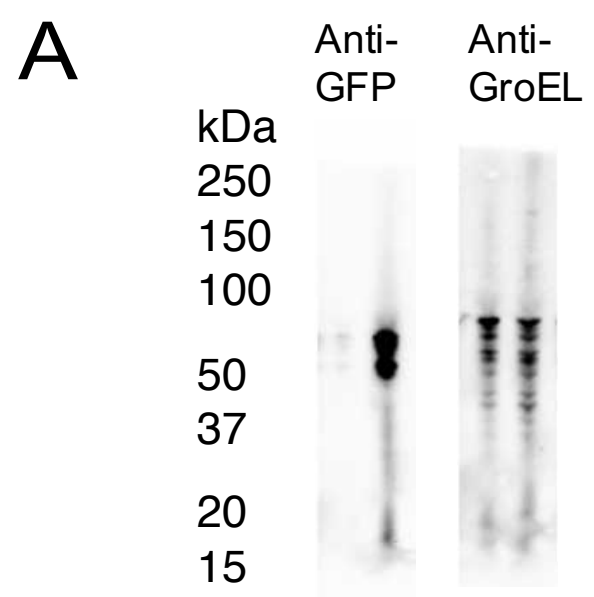

\section{ESX-3 Size Exclusion Profile}

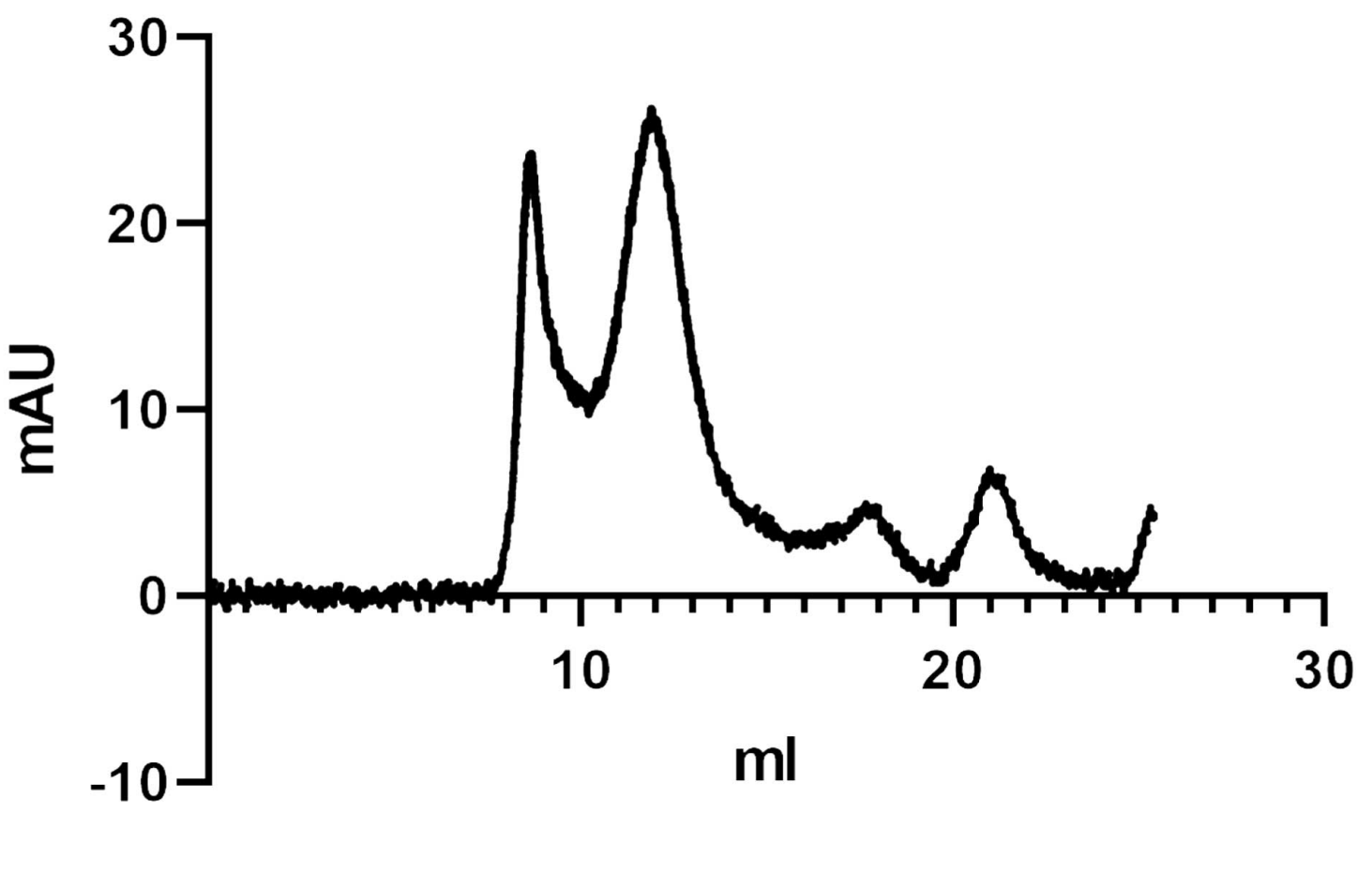

242 Fig. S2. ESX-3 expression and purification

243 (A) Western blot of solubilized cell material with EccE3 tagged with GFP. Lane 1, wild type

244 background. Lane 2, $\Delta \mathrm{IdeR}$ background. (B) Size exclusion profile for the ESX-3 purification. 

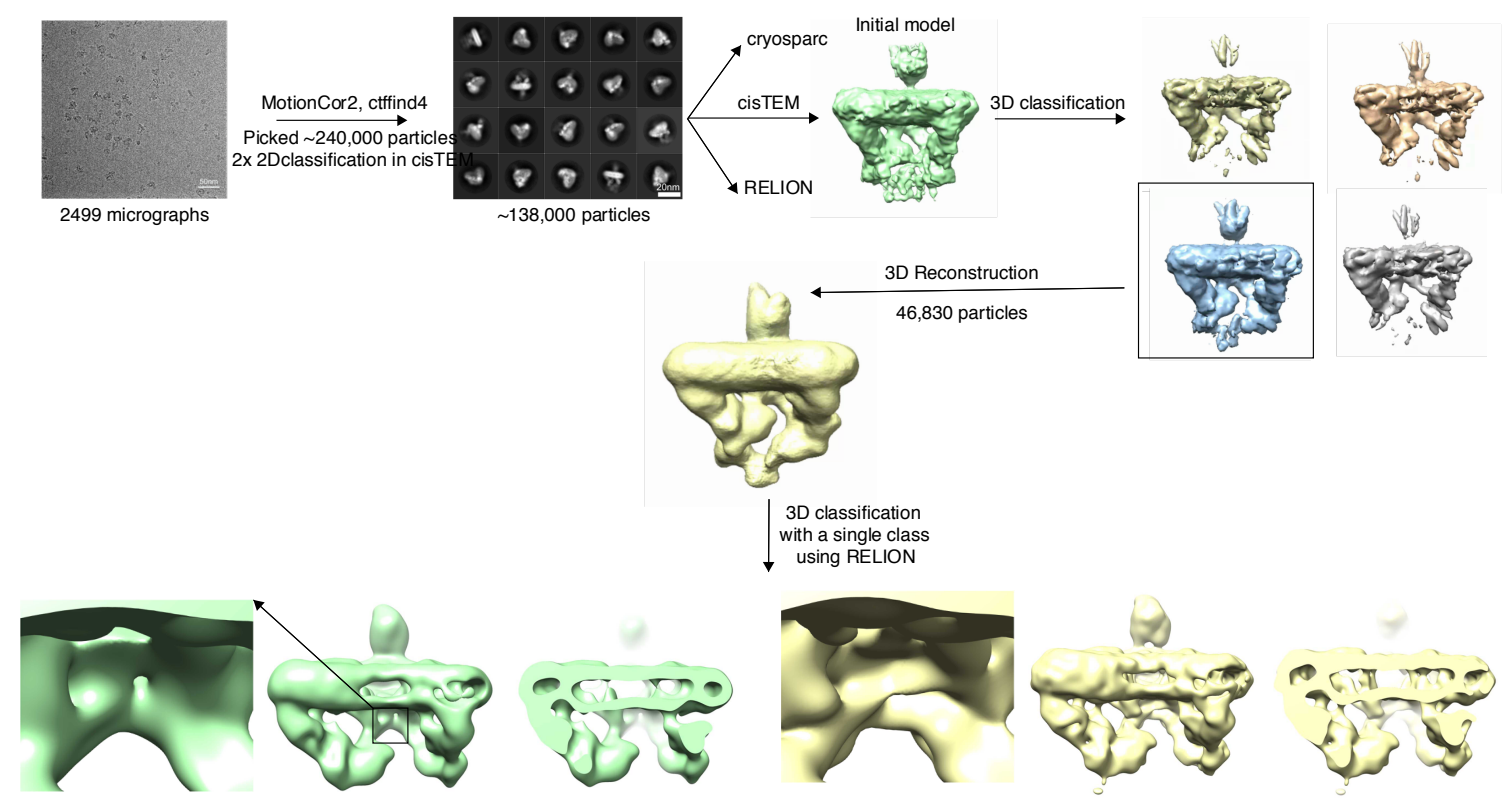

Tau2_fudge $=4$, Angular sampleing $=7.5^{\circ}$

Tau2_fudge $=8$, Angular sampleing $=3.7^{\circ}$

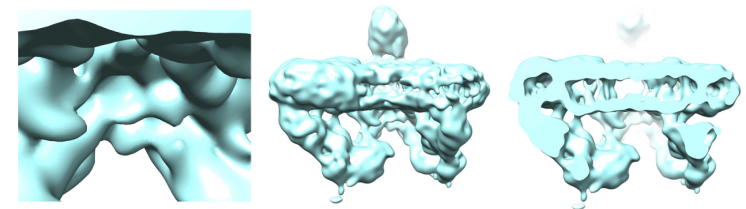

Tau2 $\_$fudge $=10$, Angular sampleing $=3.7^{\circ}$

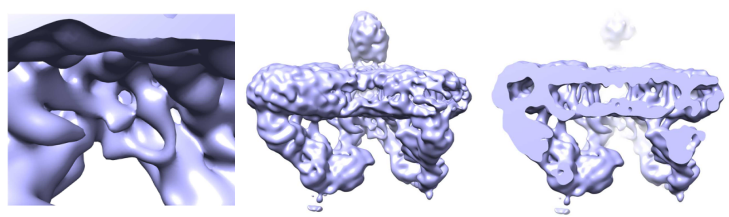

Tau2 $\_$fudge $=12$, Angular sampleing $=3.7^{\circ}$

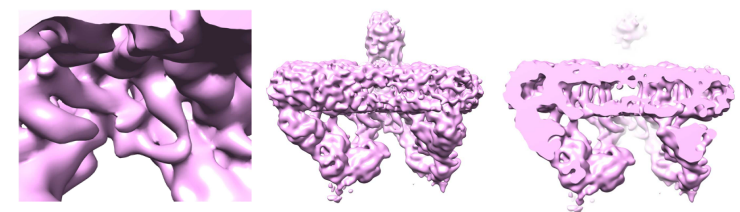

Tau2_fudge $=14$, Angular sampleing $=1.8^{\circ}$

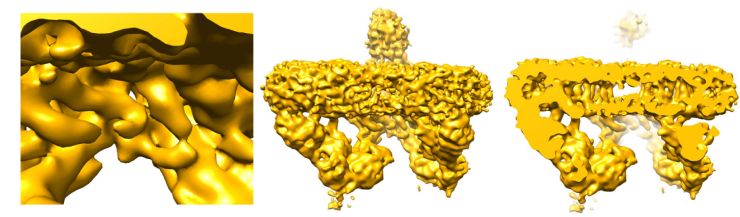

Tau2_fudge $=18$, Angular sampleing $=1.8^{\circ}$
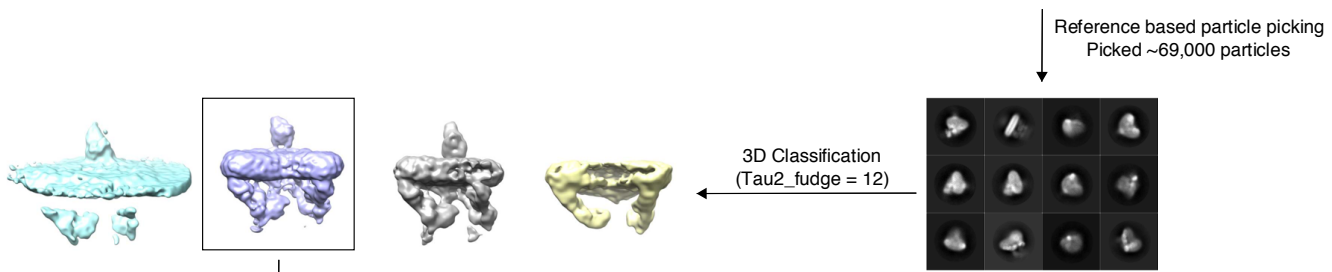

3D refinement 22,505 particle@
$\sim 4.7 \AA ̊$ resolution
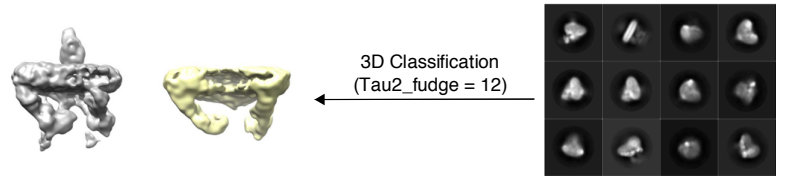

2D Classification $\sim 54,000$ particles

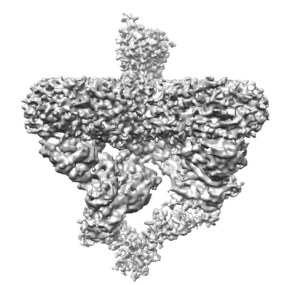

246 Fig. S3. Initial data collection and initial model generation

247 An initial data set was collected on a Talos arctica microscope. The final refinement from this data processing was used as the starting model for future data processing 

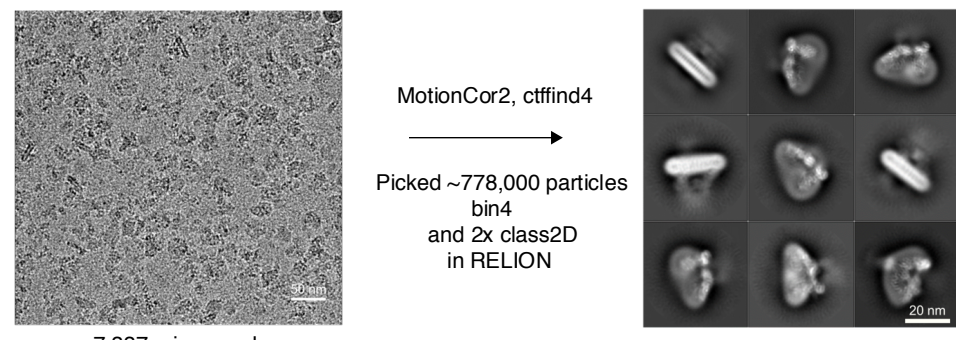

Final model from arctica collection

7,337 micrographs
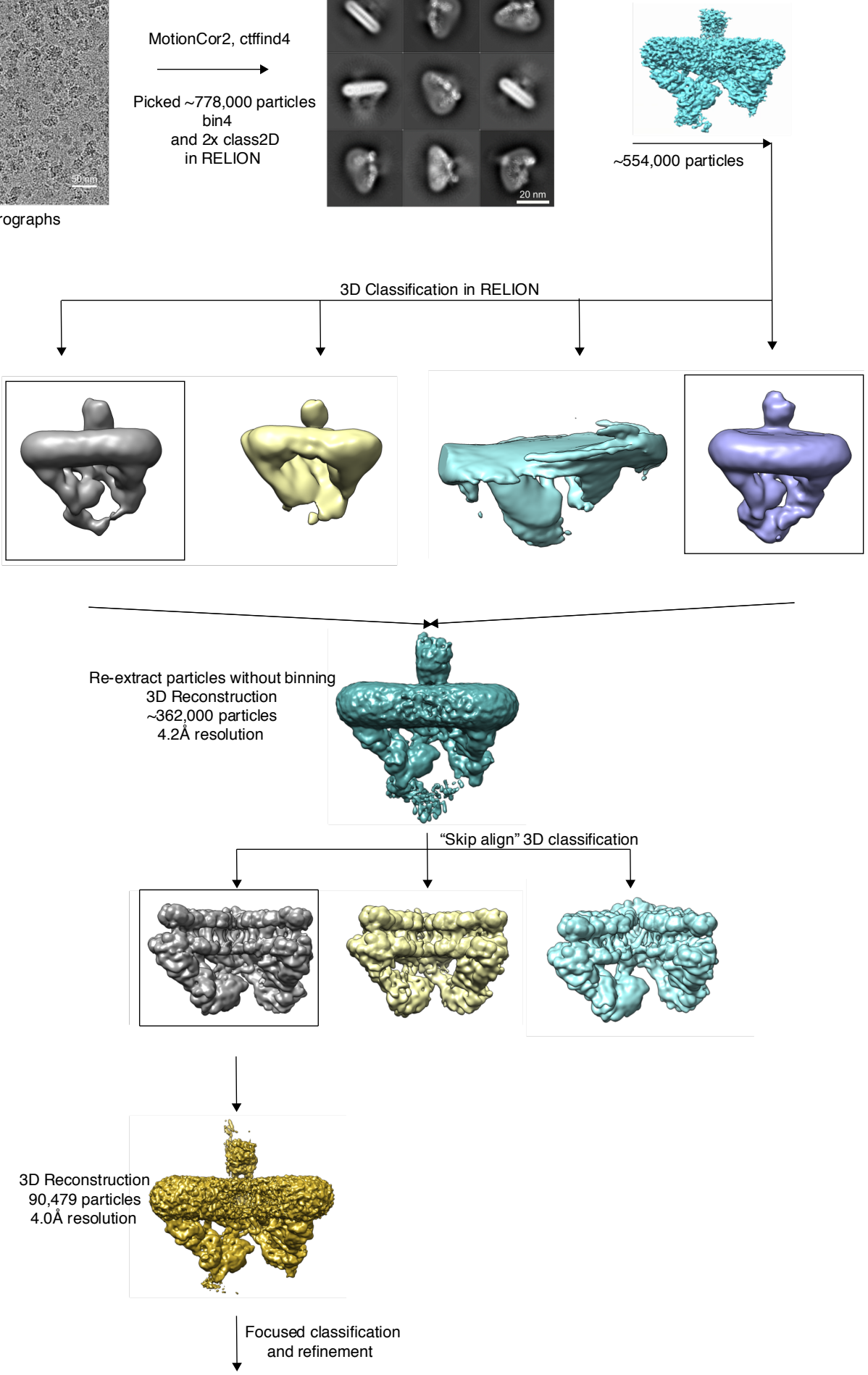

250 Fig. S4. Data processing workflow for final data collection.

251 Data was collected on a Titan Krios. Particles from the final reconstruction were subsequently used for focused classification and refinement. 

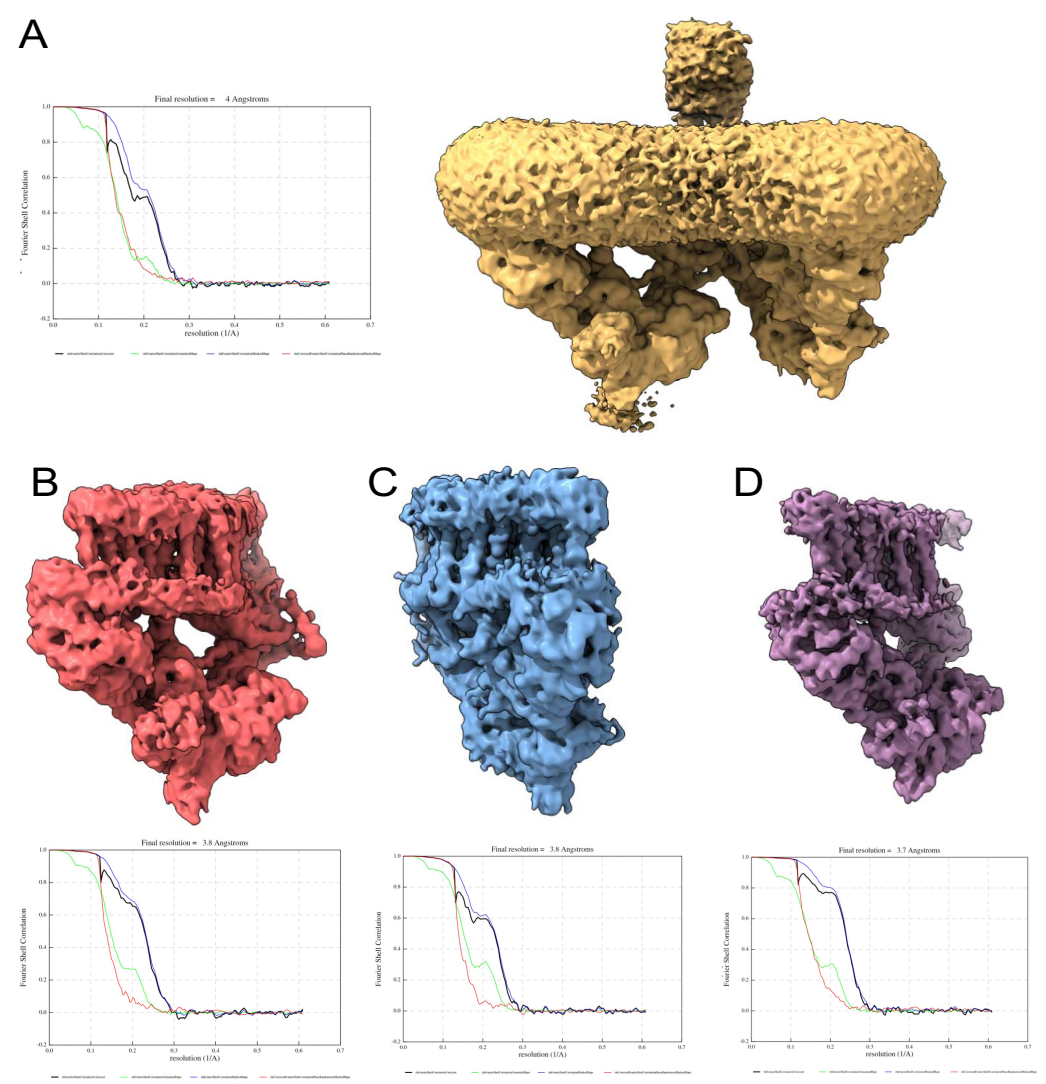

$E$

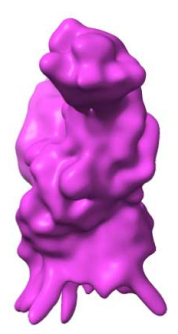

\section{$\mathrm{F}$}
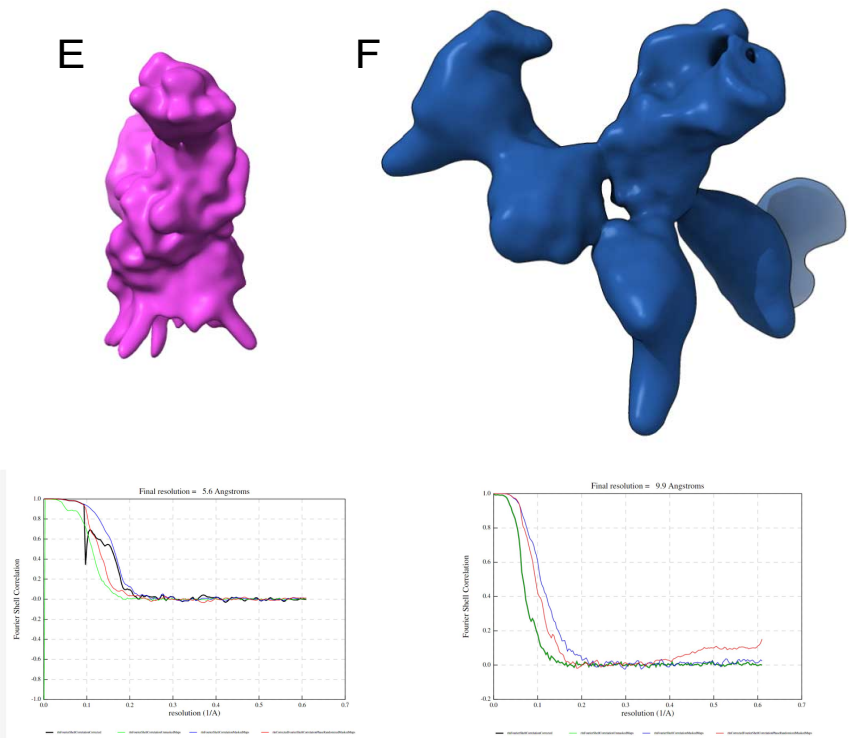

Fig. S5. Consensus and focused refinements

(A) Consensus refinement map and FSC curve for the ESX-3 complex. Focused refinement maps and FSC curves for (B) the left protomer of the ESX-3 complex. (C) the right protomer of the ESX-3 complex (D) a symmetry expanded protomer (E) the periplasmic domain (F) the lower ATPase domains of EccC. All maps are unsharpened. 

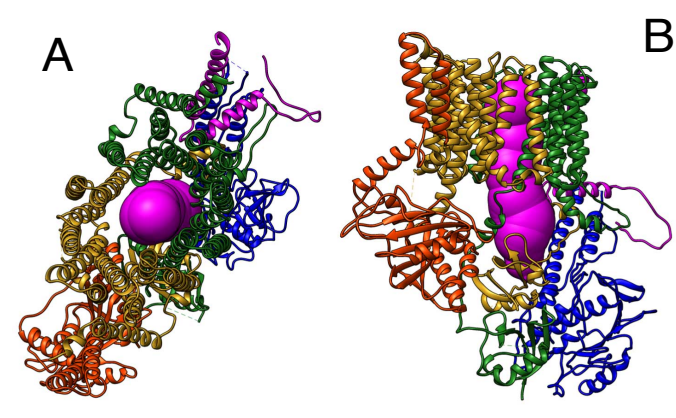

B
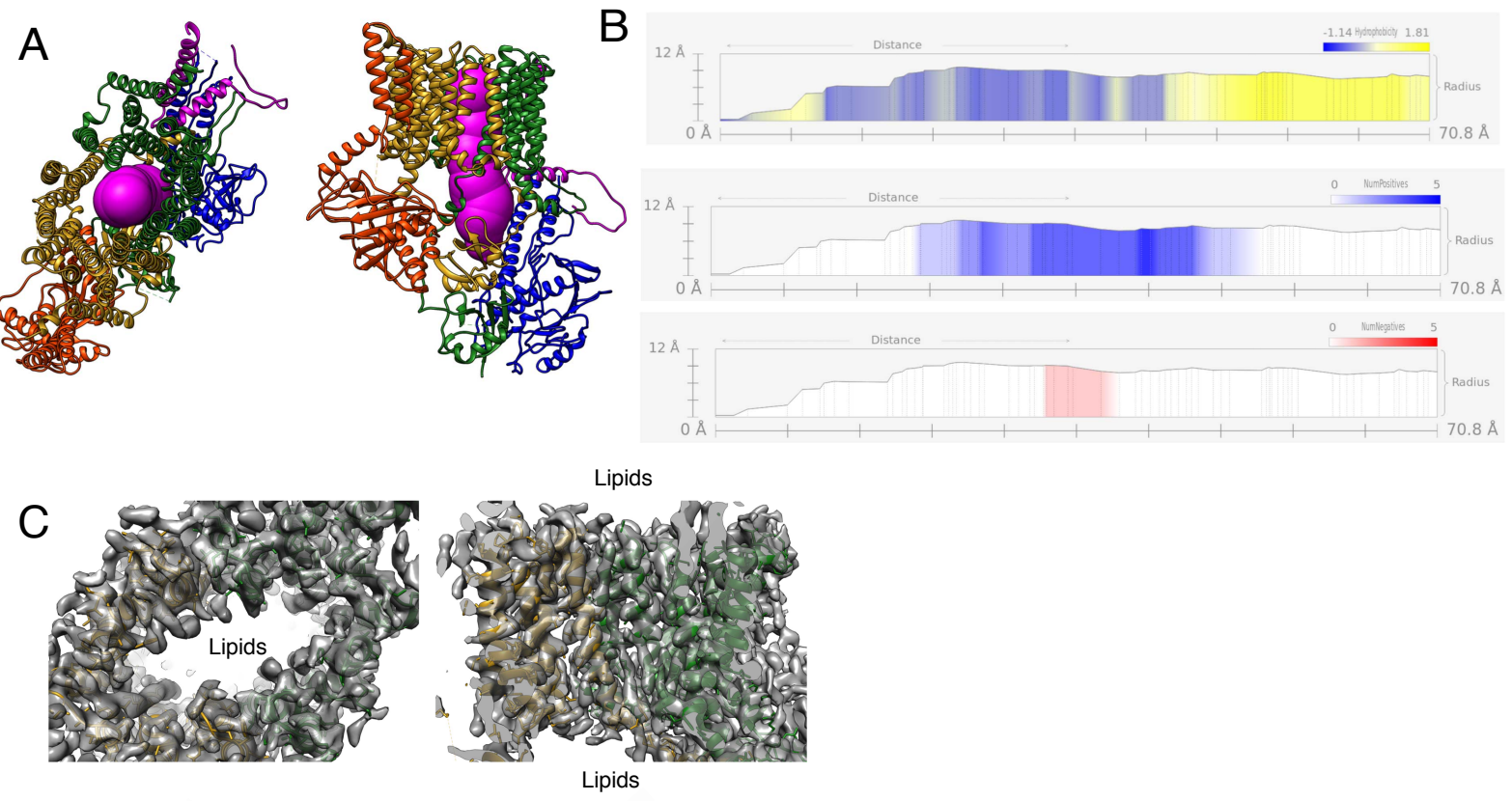

261 Fig. S6. The translocon pore

262 (A) The path of the pore as mapped by the program MOLE shown in purple spheres through one 263 protomer. (B) The path of the pore shows no constriction and positively charged at the 264 cytoplasmic face. (C) Lipid and detergent line the inside of the pore. 

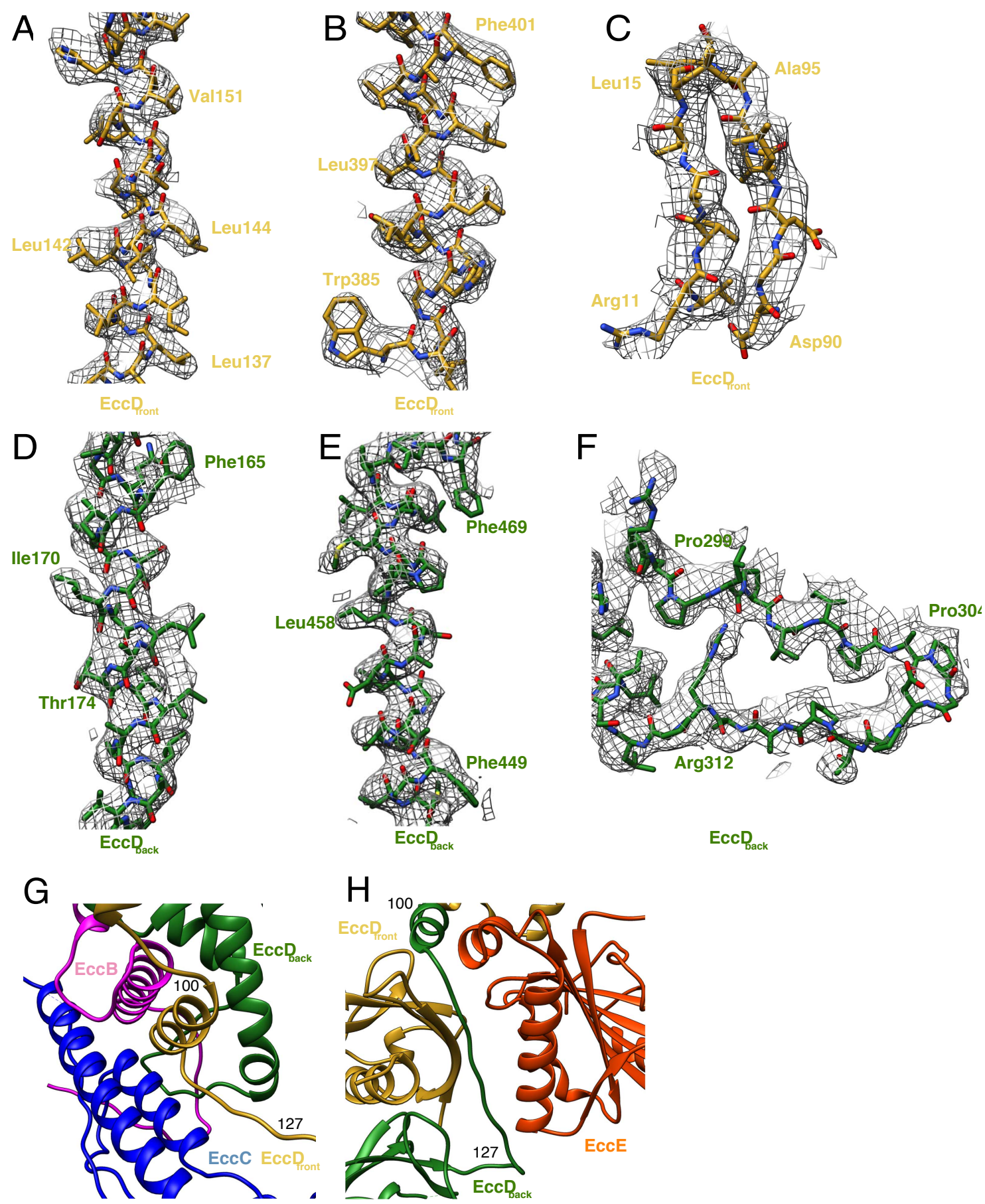

Fig. S7. EccD 3 map and model

Map to model fits for EccD front for (A) transmembrane $\alpha$-helix 1, amino acids 136-153, (B) transmembrane $\alpha$-helix 1 , amino acids 385-402, (C) soluble domain $\beta$-strands amino acids 10-15 and 90-96. Map to model fits for EccD back for (D) transmembrane $\alpha$-helix 1, amino acids 163181 (E) transmembrane $\alpha$-helix 11, amino acids 446-469 (F) soluble loop, amino acids 297-315.

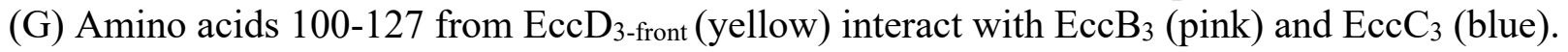
(H) Amino acids 100-127 of EccD 3 -back (green), in a distinct conformation, interact with $\mathrm{EccE}_{3}$

274 (orange) and $\mathrm{EccD}_{3 \text {-front }}$ (yellow) 

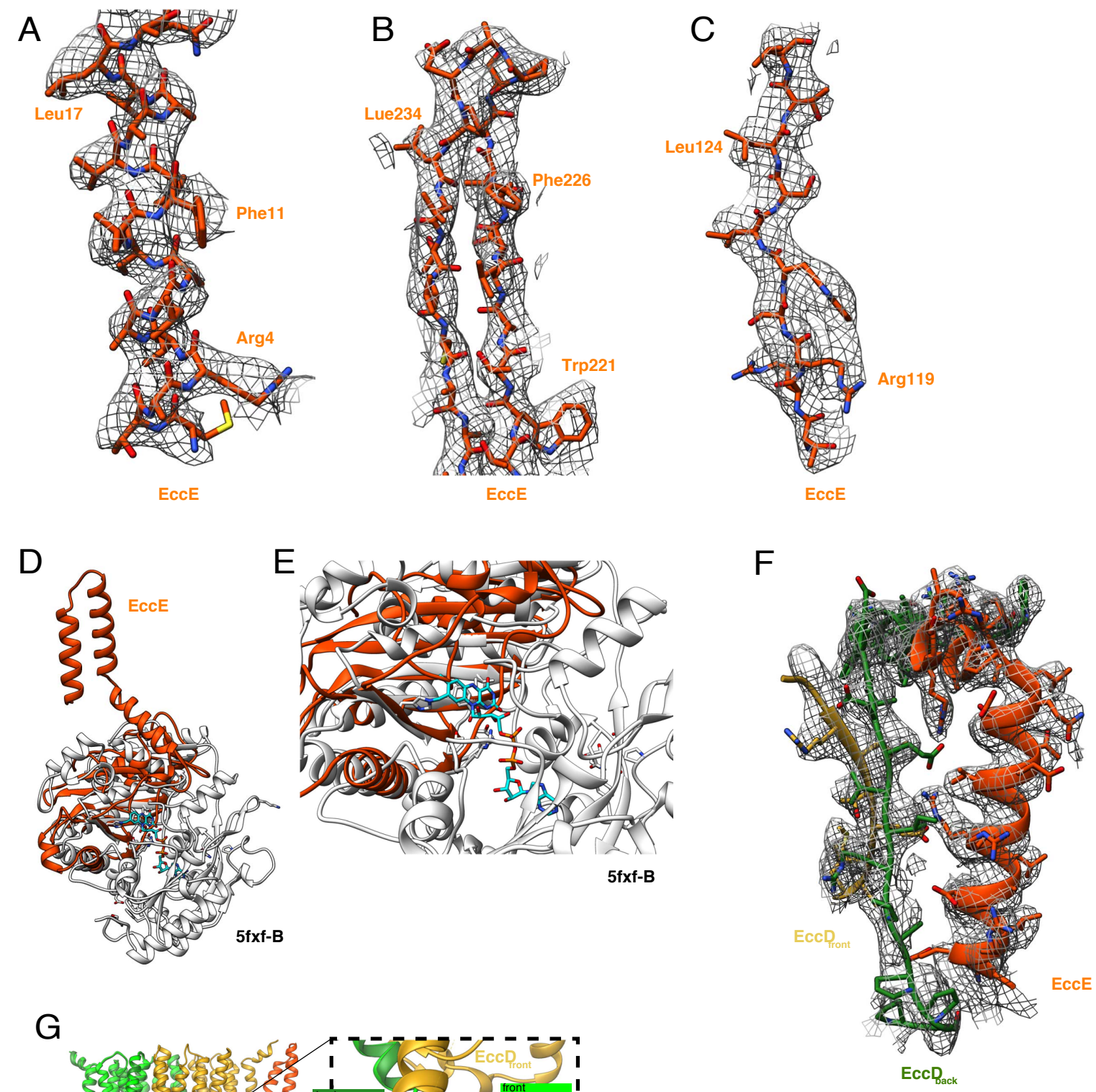

G

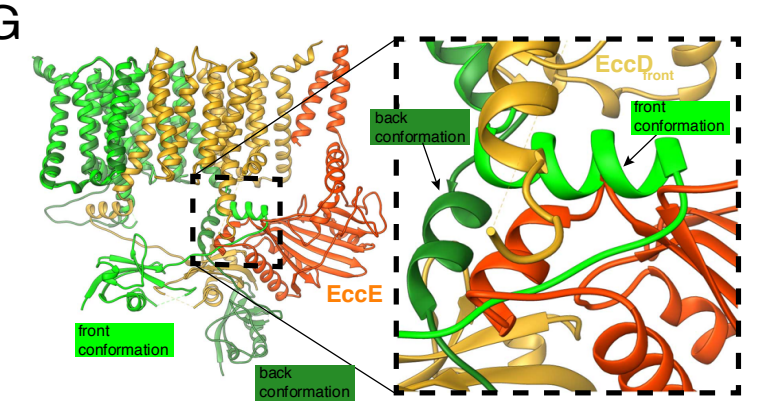

276 Fig. S8. EccE 3 map and model

277 (A) Map and model comparison for transmembrane helix 1, amino acids 1 to 18. (B) Beta strand separation, amino acids amino acids 221 to 240. (C) a single beta strand, amino acids 117 to 126 . (D) Comparison between the structure of $\mathrm{EccE}_{3}$ and the top DALI hit $5 \mathrm{fxf}-\mathrm{B}$. (E) Close up on the FADH (cyan) binding pocket of $5 \mathrm{fxf}-\mathrm{B}$ which is not present in $\mathrm{EccE}_{3}$. (F) Residues 133-163 of

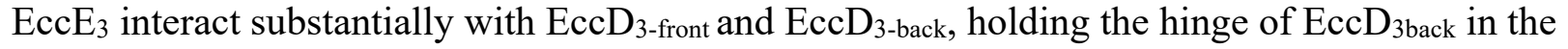
extended confromation $(\mathrm{G}) \mathrm{EccE}_{3}$ (orange) stabilizes the extended hinge conformation of $\mathrm{EccD}_{3}$ back (green) and sterically hinders it from adopting the bent conformation (neon green). 

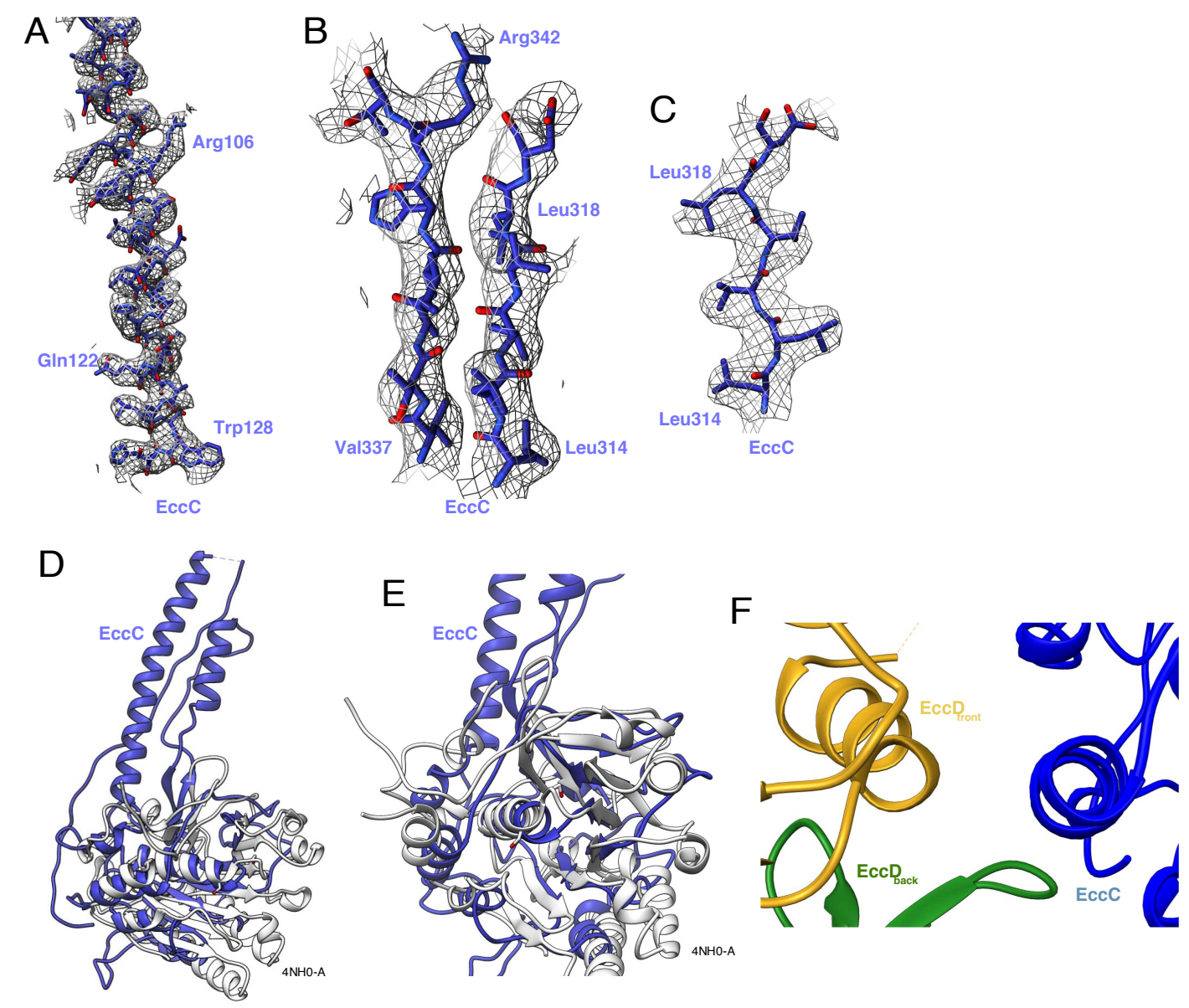

G

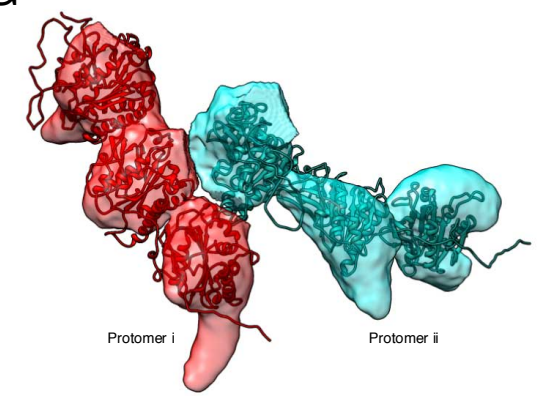

Fig. S9. EccC 3 map and model

(A) Map and model of the soluble helix of the EccC pseudo ATPase domain, amino acids amino acids 97 to 130. (B) Two beta strands in the EccC pseudo ATPase domain, amino acids 314 to 319 and 337 to 343. (C) Beta strand in the EccC pseudo ATPase domain, amino acids 314 to 319. (D) Overlay of the model of the EccC pseudo ATPase domain with the Top Dali hit, 4NH0A (white). (E) Close up of the ATP binding pocket of 4NH0-A. While the fold remains intact in the pseudo ATPase domain, the amino acids required for ATP binding and hydrolysis are mutated. (F) The protein binding domain of $\mathrm{EccC}_{3}$ interacts with the cytoplasmic domain of $\mathrm{EccD}_{3 \text {-front }}$ (yellow). (G) ATPase 1,2, and 3 domains of $\mathrm{EccC}_{3}$ with homology models docked in. 

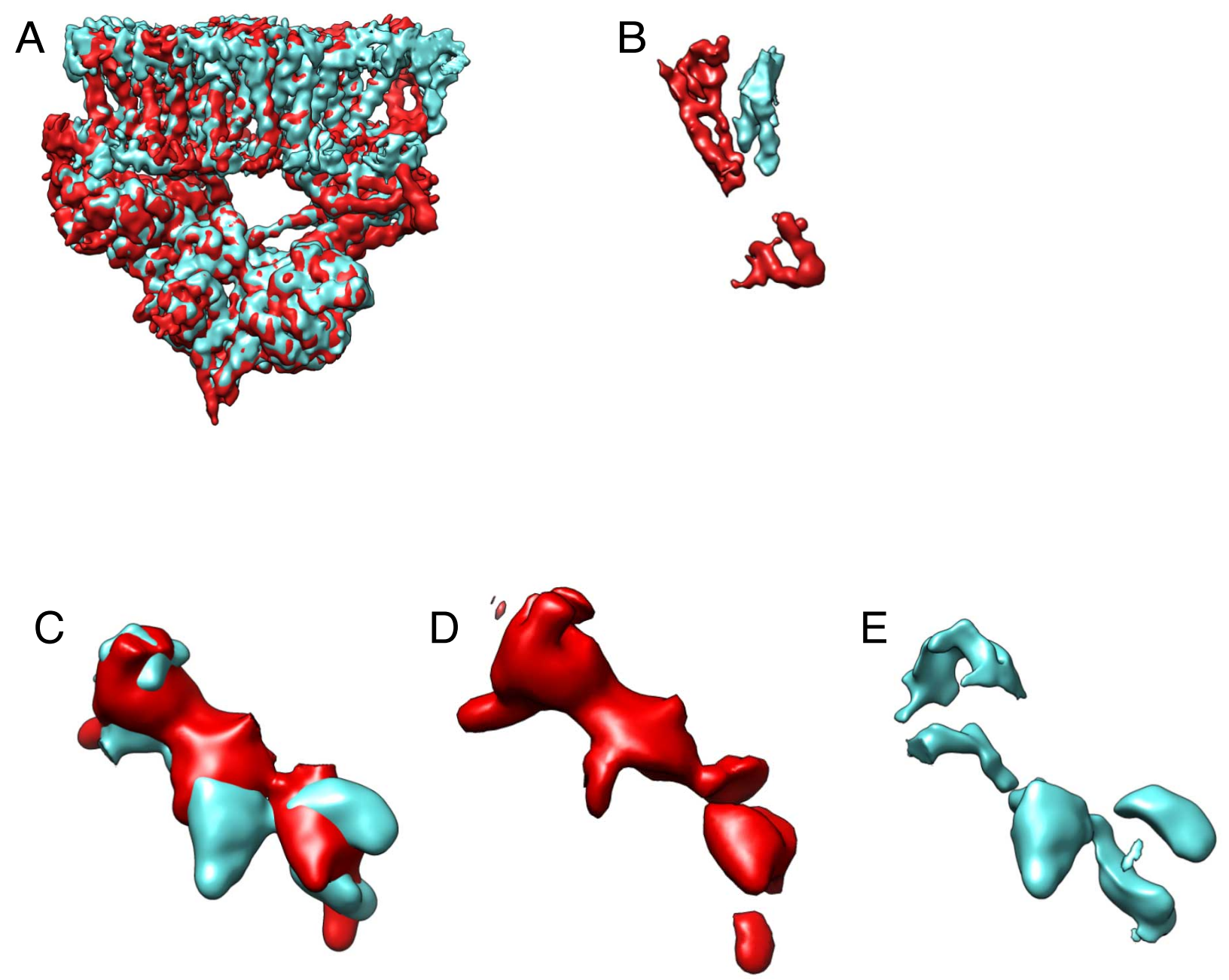

Fig. S10. Conformational differences between protomer $\mathrm{i}$ and protomer ii

(A) Overlay of the focused refined maps of the transmembrane and upper cytoplasmic regions of protomer i (red) and protomer ii (blue). The two protomers are nearly identical except in the transmembrane domains of $\mathrm{EccC}_{3}$ and the N-terminal tail of $\mathrm{EccB}_{3}$. (B) Density subtraction of the two protomers in A highlights the regions of difference. (C) Overlay of the ATPase 1,2, and 3 domains of EccC3 from protomer i (red) and protomer ii (blue). (D) Density subtraction of protomer ii from protomer i (E) Density subtraction of protomer i from protomer ii. The lack of overlap between the ATPase domains from the two protomers suggests a different conformation. 

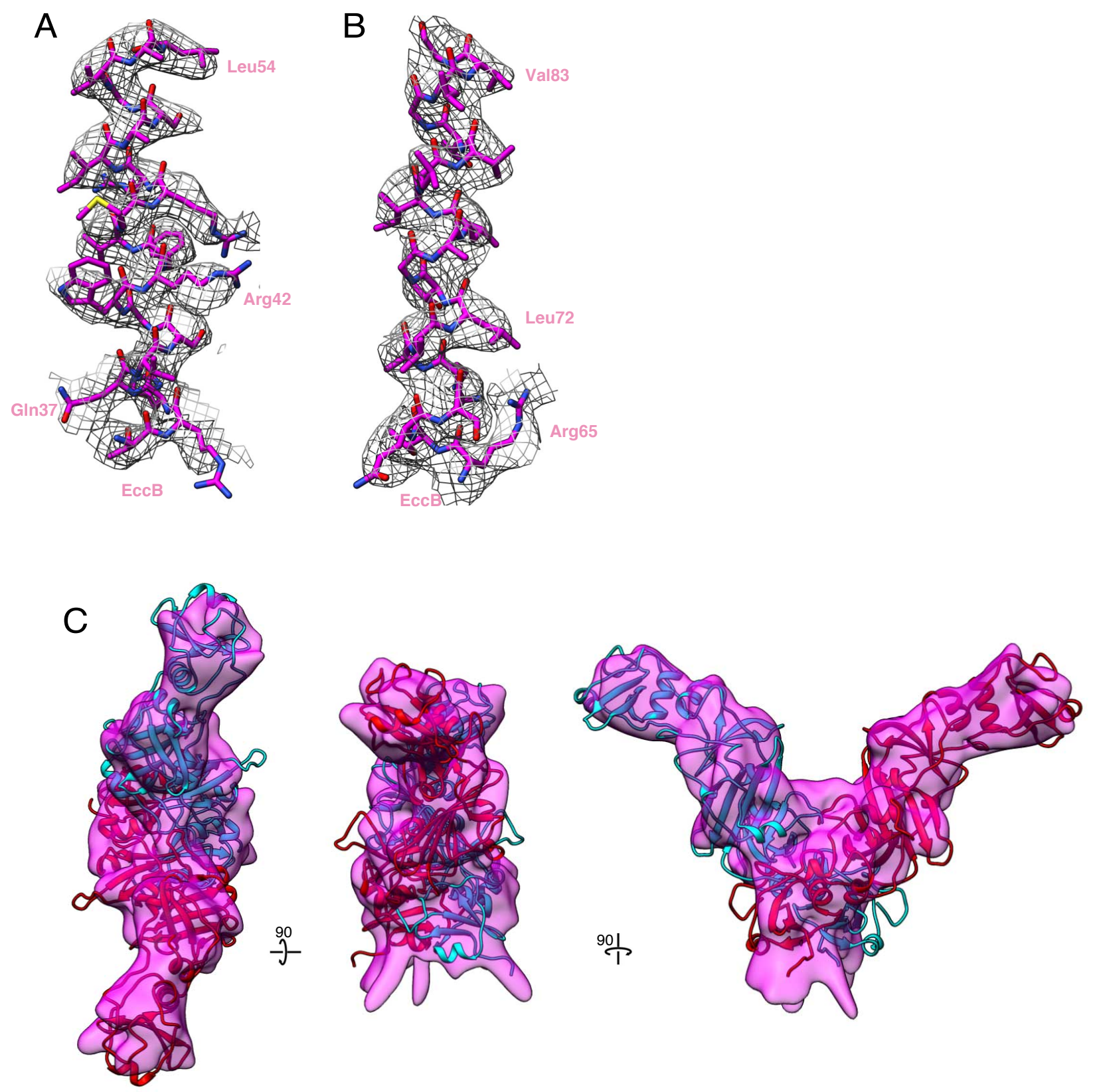

Fig. S11. EccB 3 maps and models

(A) Fit between map and model in the linker $\alpha$-helix, amino acids 34 to 54. (B) Fit between map and model in the transmembrane helix, amino acids 65 to 85. (C) Fit between homology models of the soluble domain of $\mathrm{EccB}_{3}$ and the periplasmic focused refinement map. 


\begin{tabular}{|c|c|c|c|}
\hline \multicolumn{4}{|c|}{ Data Collection } \\
\hline Collection & Initial Screening & Collection 1 & Collection 2 \\
\hline Microscope & Talos Arctica & Titan Krios & Titan Krios \\
\hline Voltage (kV) & 200 & 300 & 300 \\
\hline Detector & Gatan K2 & Gatan K2 & Gatan K2 \\
\hline Pixel size $(\AA /$ pixel $)$ & 1.14 & 0.82 & 0.82 \\
\hline Exposure Time (s) & 9 & 10 & 10 \\
\hline Electron dose $\left(\mathrm{e}-/ \AA^{2}\right)$ & 63 & 80 & 67 \\
\hline Defocus range $(\mu \mathrm{m})$ & $1.5-2.5$ & $0.4-1.2$ & $0.6-1.4$ \\
\hline Number of micrographs & 2499 & 2705 & 4632 \\
\hline \multicolumn{4}{|c|}{ Consensus Reconstruction } \\
\hline Data Set & Initial Screening & Collection $1 \& 2$ & \\
\hline Software & $\begin{array}{l}\text { Relion } 2.1 \text {, cisTEM, and } \\
\text { cryosparc }\end{array}$ & Relion 3.0 & \\
\hline$\#$ of particles, picked & 240,000 & 778,149 & \\
\hline \# of particles post, Class2D & 138,000 & 554,901 & \\
\hline \# of particles post, Class3D & 46,830 & 362,438 & \\
\hline $\begin{array}{c}\text { \# of particles post, skip align } \\
\text { Class } 3 \mathrm{D}\end{array}$ & NA & 90,479 & \\
\hline Symmetry & $\mathrm{C} 1$ & $\mathrm{C} 1$ & \\
\hline Map sharpening B-factor $\left(\AA^{2}\right)$ & NA & -160 & \\
\hline Final resolution $(\AA)$ & $4.7 \AA$ & $4.0 \AA$ & \\
\hline \multicolumn{4}{|c|}{ Focused Refinements } \\
\hline Location of focus & \# of particles & Reconstruction resolution $(\AA)$ & \\
\hline Protomer i & 76,967 & 3.8 & \\
\hline Protomer ii & 66,239 & 3.7 & \\
\hline Symmetry expanded protomer & 52,067 & 3.7 & \\
\hline Periplasmic EccB & 70,000 & 5.5 & \\
\hline ATPase 1,2 , and 3 & 30,000 & 10 & \\
\hline
\end{tabular}

\section{Table S1 Data collection and refinement statistics}

312 Collection parameters for initial test data set on the Talos Arctica and final collection on Titan

313 Krios microscopes. Refinement details for initial model, consensus map, and focused

314 refinements 


\section{Model Refinement}

Total amino acids

3,156

All-atom clashscore

13.49

Ramachandran outliers

$0.00 \%$

Ramachandran allowed

$5.39 \%$

Ramachandran favored

$94.61 \%$

Rotamer Outliers

$0.00 \%$

Cbeta Deviations

$0 \%$

Cis-proline

$0 \%$

Cis-general

$0.00 \%$

Twisted proline

$0 \%$

Twisted general

$0.00 \%$

\section{6}

\section{Table S2 Model Refinement Statistics.}

318 Final refinement validation for all amino acids which were modeled de novo (i.e. the translocon

319 pore and translocon gate). 


\begin{tabular}{|c|c|c|c|c|c|c|c|}
\hline \multicolumn{8}{|c|}{ Top Dali Server Hits } \\
\hline Hit Rank & PDB Chain ID & Z score & RMSD & lali & nres & $\%$ id & Description \\
\hline \multicolumn{8}{|c|}{$\mathrm{EccB}_{3}$} \\
\hline 1 & 6kre-b & 2.7 & 5.8 & 59 & 444 & 7 & T-complex protein 1 subunit alpha \\
\hline 2 & None & NA & NA & NA & NA & NA & NA \\
\hline \multicolumn{8}{|c|}{$\mathrm{EccC}_{3}$} \\
\hline 1 & $4 \mathrm{nh} 0-\mathrm{A}$ & 14.8 & 3.3 & 207 & 861 & 16 & Cell division FtsK/SpoIIE (EccC) \\
\hline 2 & 2iuu-A & 14.5 & 4.9 & 198 & 408 & 16 & DNA translocase FtsK \\
\hline 3 & 4nla-A & 13.9 & 3.4 & 205 & 555 & 18 & Cell division FtsK/SpoIIE \\
\hline 4 & 4lya-A & 12.8 & 3.4 & 198 & 521 & 14 & EssC \\
\hline 5 & $5 \mathrm{fv} 0-\mathrm{A}$ & 11.9 & 3.2 & 182 & 508 & 13 & EssC \\
\hline \multicolumn{8}{|c|}{$\mathrm{EccD}_{3}$} \\
\hline 1 & $4 \mathrm{kv} 2-\mathrm{A}$ & 9.9 & 1.6 & 70 & 90 & 30 & ESX-1 Secretion system protein EccD $_{1}$ \\
\hline 2 & $3 q k y-A$ & 9.3 & 10.3 & 138 & 251 & 7 & Outer membrane assembly lipoprotein (BamD) \\
\hline 3 & $6 \mathrm{e} 9 \mathrm{v}-\mathrm{J}$ & 9.2 & 7.2 & 156 & 211 & 10 & Dhf79 filament \\
\hline 4 & $3 j 99-G$ & 9.2 & 8.3 & 150 & 286 & 7 & Vesicle-fusing ATPase \\
\hline 5 & 5wfu-A & 9.1 & 5.8 & 119 & 234 & 3 & 14-3-3 protein beta/alpha \\
\hline \multicolumn{8}{|c|}{$\mathrm{EccE}_{3}$} \\
\hline 1 & $5 f x f-B$ & 7.5 & 7.1 & 184 & 525 & 6 & Eugenol oxidase \\
\hline 2 & $516 \mathrm{~g}-\mathrm{A}$ & 7.1 & 6.6 & 168 & 473 & 9 & FAD linked oxidase-like protein \\
\hline 3 & $2 \mathrm{axr}-\mathrm{A}$ & 7.0 & 7.0 & 166 & 479 & 10 & Glucooligosaccharide oxidase \\
\hline 4 & $6 f 73-B$ & 7.0 & 7.9 & 164 & 548 & 12 & MTVA0615 \\
\hline 5 & $4 p 52-A$ & 6.6 & 11.8 & 98 & 315 & 8 & Homoserine kinase \\
\hline
\end{tabular}

\section{Table S3 Top Dali server hits}

322 Top 5 non-redundant hits from the Dali server compared against the full PDB for the de novo

323 built sections of $\mathrm{EccB}_{3}, \mathrm{EccC}_{3}, \mathrm{EccD}_{3}$, and $\mathrm{EccE}_{3}$. 


\begin{tabular}{|c|c|c|}
\hline \multicolumn{3}{|c|}{ Interaction interfaces } \\
\hline Monomer 1 & Monomer 2 & Burried surface area (units) \\
\hline $\mathrm{EccD}_{\text {front }}$ - protomer i & EccD back - protomer i & 3154 \\
\hline EccD $_{\text {front }}$ - protomer ii & EccD back - protomer ii & 3116.3 \\
\hline EccD back - protomer i & EccB - protomer i & 1370.2 \\
\hline EccD back - protomer ii & EccB - protomer ii & 1295.6 \\
\hline EccE - protomer i & $\mathrm{EccD}_{\text {front }}$ - protomer i & 1157.2 \\
\hline EccE - protomer ii & EccD $D_{\text {front }}$ - protomer ii & 1122.4 \\
\hline Ecc $D_{\text {back }}$ - protomer $\mathrm{i}$ & EccC - protomer i & 881.9 \\
\hline EccD back - protomer ii & EccC - protomer ii & 825.1 \\
\hline EccD front $_{\text {- protomer } \mathrm{i}}$ & EccC - protomer i & 779.9 \\
\hline EccD $_{\text {front }}$ - protomer ii & EccC - protomer ii & 746.6 \\
\hline EccB - protomer i & EccC - protomer i & 635.7 \\
\hline EccE - protomer i & EccD back - protomer i & 608.5 \\
\hline EccE - protomer ii & EccD $_{\text {back }}$ - protomer ii & 600.1 \\
\hline EccB - protomer i & EccC - protomer ii & 576.1 \\
\hline EccB - protomer ii & EccC - protomer ii & 502.3 \\
\hline EccB - protomer i & EccD back - protomer ii & 482.1 \\
\hline EccB - protomer i & $\mathrm{EccD}_{\text {front }}$ - protomer i & 297.2 \\
\hline EccB - protomer ii & EccD front - protomer ii & 288.6 \\
\hline EccB - protomer i & EccB - protomer ii & 255.6 \\
\hline EccC - protomer i & EccC - protomer ii & 213.6 \\
\hline EccB - protomer i & EccD $_{\text {front }}$ - protomer ii & 168.9 \\
\hline EccD back - protomer i & EccB - protomer ii & 56.6 \\
\hline EccD back - protomer i & EccD back - protomer ii & 49.9 \\
\hline EccD back - protomer i & EccC - protomer ii & 40.1 \\
\hline \multicolumn{3}{|c|}{ Cross-protomer interaction interfaces } \\
\hline EccB - protomer i & EccB, EccC, EccD - protomer ii & 1482.7 \\
\hline EccC - protomer i & EccC - protomer ii & 213.6 \\
\hline Ecc $D_{\text {back }}$ - protomer $\mathrm{i}$ & $\mathrm{EccB}, \mathrm{EccC}, \mathrm{EccD}_{\text {back }}$ - protomer ii & 146.6 \\
\hline
\end{tabular}

\section{Table S4 Buried surface area.}

328 Buried surface area between subunits as measured by PISA. 
331 1. S. Q. Zheng et al., MotionCor2: anisotropic correction of beam-induced motion for improved cryo-electron microscopy. Nature Methods 14, 331 (2017).

2. A. Rohou, N. Grigorieff, CTFFIND4: Fast and accurate defocus estimation from electron micrographs. J Struct Biol 192, 216-221 (2015).

3. J. Zivanov et al., New tools for automated high-resolution cryo-EM structure determination in RELION-3. eLife 7, e42166 (2018).

4. T. Grant, A. Rohou, N. Grigorieff, cisTEM, user-friendly software for single-particle image processing. eLife 7, e35383 (2018).

5. A. Punjani, J. L. Rubinstein, D. J. Fleet, M. A. Brubaker, cryoSPARC: algorithms for

6. E. F. Pettersen et al., UCSF Chimera-A visualization system for exploratory research and analysis. Journal of Computational Chemistry 25, 1605-1612 (2004).

7. P. Emsley, B. Lohkamp, W. G. Scott, K. Cowtan, Features and development of Coot. Acta Crystallographica Section D 66, 486-501 (2010).

8. M. Källberg et al., Template-based protein structure modeling using the RaptorX web server. Nature Protocols 7, 1511 (2012).

9. P. V. Afonine et al., Real-space refinement in PHENIX for cryo-EM and crystallography. Acta Crystallographica Section D 74, 531-544 (2018).

10. L. G. Trabuco, E. Villa, E. Schreiner, C. B. Harrison, K. Schulten, Molecular dynamics flexible fitting: A practical guide to combine cryo-electron microscopy and X-ray crystallography. Methods 49, 174-180 (2009).

11. R. T. Kidmose et al., Namdinator - Automatic Molecular Dynamics flexible fitting of structural models into cryo-EM and crystallography experimental maps. bioRxiv, 501197 (2018).

12. L. Pravda et al., MOLEonline: a web-based tool for analyzing channels, tunnels and pores (2018 update). Nucleic Acids Research 46, W368-W373 (2018).

13. E. Krissinel, Stock-based detection of protein oligomeric states in jsPISA. Nucleic Acids Research 43, W314-W319 (2015).

14. L. Holm, Benchmarking fold detection by DaliLite v.5. Bioinformatics, (2019).

15. T. D. Goddard et al., UCSF ChimeraX: Meeting modern challenges in visualization and analysis. Protein Science 27, 14-25 (2018). 